Pacific Journal of Mathematics

RIGHT DERIVATIONS AND RIGHT DIFFERENTIA 


\title{
RIGHT DERIVATIONS AND RIGHT DIFFERENTIAL OPERATORS
}

\author{
Moss E. SWeEdLeR
}

Dedicated to Gerhard Hochschild on the occasion of his 65th birthday

In the beginning are defined right differential operators for noncommutative algebras. Differential operators give rise to right derivations, jet and Kähler modules for noncommutative algebras, differential separability and differential inseparability (called differentially stacked). Differential separability is related to the abelianized algebra being separable, Differentially stacked is related to pure inseparability of noncommutative algebras.

Introduction. Suppose $A$ is a not necessarily commutative algebra with right $A$-modules $M, N$. For $f \in \operatorname{Hom}(M, N), a \in A$, $m \in M$ let $[f, a] \in \operatorname{Hom}(M, N)$ be defined by $[f, a](m) \equiv f(m a)-f(m) a$. So $\operatorname{Hom}_{A}(M, N)=\{f \in \operatorname{Hom}(M, N) \mid[f, \alpha]=0$ for all $a \in A\}$. Inductively define $\mathscr{D}_{A}^{0}(M, N)=\operatorname{Hom}_{A}(M, N)\left(\right.$ or $\left.\mathscr{D}_{A}^{-1}(M, N)=\{0\}\right)$ and

$$
\mathscr{D}_{A}^{n}(M, N)=\left\{f \in \operatorname{Hom}(M, N) \mid[f, a] \in \mathscr{D}_{A}^{n-1}(M, N) \text { for all } a \in A\right\} \text {. }
$$

$\mathscr{D}_{A}^{n}(M, N)$ is the module of $n$th order right differential operators from $M$ to $N$. This follows the definition in [3, (2.1.1) p.210] where $A$ was assumed commutative.

An important example of first order right differential operator is the right derivation, a linear map $d: A \rightarrow N$ satisfying $d(\alpha \alpha)=$ $d(\alpha) \alpha+d(\alpha) a . \quad \mathscr{D}_{A}^{1}(A, N)$ splits up as a direct sum of $\operatorname{Hom}_{A}(A, N)=N$ and the module of right derivations from $A$ to $N$. More about right derivations in a while.

As in the commutative case $\mathscr{D}_{A}^{n}(M,-)$ has a universal representing object. A right $A$-module $J_{n}(M)$ together with $j_{n} \in \mathscr{D}_{A}^{n}\left(M, J_{n}(M)\right)$ where for each $f \in \mathscr{D}_{A}^{n}(M, N)$ there is a unique $F \in \operatorname{Hom}_{A}\left(J_{n}(M), N\right)$ with $F j_{n}=f$. The key to dropping the commutativity requirement on $A$ is the introduction of the opposite algebra $\bar{A}$ and the enveloping algebra $A^{e}=\bar{A} \otimes A$. A fair amount of our first section is an adaptation of $[3, \S 2$, p. 210-220] to the noncommutative, a process of discovering which $A$ 's to leave and which must become $\bar{A}$ 's.

The (jet) modules $J_{n}(M)$ depend on $Z_{n}(A)$-an $A$-bimodule-in that $J_{n}(M) \cong M \otimes_{A} J_{n}(A)$ an isomorphism preserving universal properties. $A$ is right differentially separable if for all $n$ (or $n=2$ ) $\left(j_{n}, J_{n}(A)\right) \sim(I, A)$. This is equivalent to $\mathscr{D}_{A}^{n}(M, N)=\operatorname{Hom}_{A}(M, N)$ for all $n$ and right $A$-modules $M, N$. In other words there are no interesting (non $A$-linear) right differential operators. The opposite 
extreme is when for some $n$

$$
\left(j_{n}, J_{n}(A)\right) \sim\left(A \stackrel{(a \rightarrow \overline{1} \otimes a)}{\longrightarrow} A^{e}, A^{e}\right) .
$$

In this case $A$ is called right n-differentially stacked which is strongly related to pure inseparability as shall be seen. $A$ being $n$-differentially stacked is equivalent to $\mathscr{D}_{A}{ }^{m}(M, N)=\operatorname{Hom}(M, N)$ for all $m \geqq n$ and right modules $M, N$. In other words all maps from $M$ to $N$ (linear with respect to the ground ring not $A$ ) are differential operators of order $n$.

Our second section is devoted to characterizing right differentially stacked algebras. The notion of not necessarily commutative purely inseparable algebra is defined in [5, Definition 1, p. 343]. A finite dimensional algebra over a ground field is purely inseparable if and only if mod its Jacobson radical it is a purely inseparable field extension. [7, Corollary 13, p. 353].

The main result of section two is the equivalence of the following three conditions for a finite dimensional algebra $A$ over a ground field:

(i) $A$ is a purely inseparable algebra,

(ii) $\left\{z \in A^{e} \mid z=\overline{1} \otimes a-\bar{a} \otimes 1\right.$ for $\left.a \in A\right\}$ lies in the Jacobson radical of $A^{e}$.

(iii) $A$ is right $n$-differentially stacked for some $n$.

Three and four are short sections giving the behavior of $J_{n}(A)$ with respect to algebra maps $A \rightarrow B$ and base extension.

In the sixth section right differentially separable algebras are characterized. Suppose $A$ is an algebra over ground ring $R$. Let $\mathscr{K}$ be the commutator ideal, i.e., the two-sided ideal generated by $\{x \in A \mid x=a b-b a, a, b \in A\}$ so that $A / \mathscr{K}$ is $A$ "abelianized". The main results concerning right differential separability are:

If $R$ is semi-simple and $A$ separable then $A$ is right differentially separable.

If $A$ is commutative and $A \otimes_{R} A$ Noetherian then $A$ is separable if and only if $A$ is right differentially separable.

If $A$ is right differentially separable then $A / \mathscr{K}$ is right differentially separable.

If the Kähler module of $A$ is a finitely generated $A$-module and $A / \mathscr{C}$ is right differentially separable then $A$ is right differentially separable.

If $R$ is Noetherian, $A$ contains a finitely generated subalgebra $B$ and $A$ is generated as an algebra by $B$ and the elements of $A$ which are invertible with inverse in $B$ then $A$ is right differentially separable if and only if $A / \mathscr{K}$ is separable. In particular finitely generated algebra over Noetherian rings are right differentially separable if and only if abelianized they are separable. 
Right derivations-mentioned earlier are the main tool used to characterize right differential separability. An interesting example of right derivation arises from skew commutative graded algebras such as the exterior algebra. For such an algebra, projection onto the odd graded part is a right derivation.

A Kähler module exists for right derivations, being a right $A$-module $K_{A}$ and right derivation $\partial: A \rightarrow K_{A}$ with the property that for any right $A$-module $M$ and right derivation $g: A \rightarrow M$ there is a unique right $A$-module map $G: K_{A} \rightarrow M$ with $\partial G=g$. Right differential separability of $A$ is equivalent to $K_{A}=\{0\}$. Among results about $K_{A}$ is a finite generation theorem which asserts that $K_{A}$ is a finitely generated $A$-module if $A$ is generated as an algebra by a finitely generated subalgebra $B$ and elements of $A$ having inverse in $B$. This is not a surprising result since on inverses a right derivation $d$ satisfies $d\left(a^{-1}\right)=-d(a) \cdot a^{-2}$.

Like derivations the kernel of a right derivation is a subalgebra. Unlike derivations, right derivations vanish on commutators, $[a, b]=$ $a b-b a$. Which is why $A$ "abelianized" comes into the characterization of right differential separability.

If $M$ is a right $A$-module $\operatorname{Hom}(A, M)$ is an $A$-bimodule. We show how the right derivations from $A$ to $M$ naturally correspond to a submodule of the ordinary derivations from $A$ to $\operatorname{Hom}(A, M)$ and characterize the submodule. This technique is used to consider the matter of extending a map defined on a subset of an algebra to a right derivation of the algebra.

One of the most interesting and important theorems about right derivations yields a factorization. Suppose $d: A \rightarrow M$ is a right derivation and $M=d(A) A$. If $\mathscr{C}_{d}$ is the largest ideal of $A$ lying in the subalgebra Ker $d$ then $M \mathscr{C}_{d}=\{0\}$ so that $M$ inherits an $A / \mathscr{C}_{d^{-}}$ module structure and $d$ factors

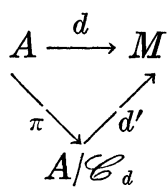

d' being a right derivation for $A / \mathscr{C}_{d} . \quad A / \mathscr{C}_{d}$ has the properties that all commutators lie in its Jacobson radical and Ker $d^{\prime}$ is a central subalgebra.

Used in showing that all commutators in $A / \mathscr{C}_{d}$ lie in the Jacobson radical is the result:

If $U$ is a simple ring with all commutators central then $U$ is commutative.

We include a snazzy proof, (5.21).

One corollary to the factorization theorem is that a semi-simple 
algebra with faithful Kähler module is commutative. In particular a simple algebra with nonzero Kähler module must be a field.

We systematically work with right differential operators and right derivations. The left analogue is mirror. Conditions (i) and (ii) of (2.1) the characterization of right differentially stacked algebras do not depend on right or left. Hence being right differentially stacked is equivalent to being left differentially stacked for finite dimensional algebras over a field. The same is true-to some extent-for characterizations of right differentially separable algebras in $\S 6$. However, in this paper we do not systematically compare right differential conditions with left differential conditions.

Thanks to Alex Rosenberg for suggesting the condition (ii) in the characterization of right differentially stacked algebras (2.1).

The most interesting order in which to read the paper is $\S 5$, $\S 6, \S 1, \S 2, \S 3, \S 4$. The logical gaps are off-set by the greater motivation and interest of the material.

1. Right differential operators, Throughout the paper $R$ is a commutative ring with identity. Unadorned $\otimes$, Hom and End denote $\otimes_{R}, \mathrm{Hom}_{R}$ and $\operatorname{End}_{R}$. Algebras are unitary $R$-algebras unless otherwise indicated. Subalgebras and algebra maps preserve unit. If $A$ is an algebra and we specify that $M$ is an $A$-module (left or right), this means that

1. $M$ is an $R$-module,

2. $M$ is a unitary $A$-module,

3. the $R$-module structue on $M$ induced by $M$ being an $A$-module agrees with the original $R$-module structure on $M$.

Thus if $M$ happens to be a module for several algebras all the underlying $R$-module structures are the same (as the original $R$-module structure on $M$ ).

Much of the treatment of differential operators in [3] generalizes to noncommutative algebras. We present the generalization giving references to [3].

For any right $A$-module $L$ and $a \in A$, let $a^{r} \in \operatorname{Hom}(L, L)$ be defined by

$$
a^{r}(l)=l a, \text { for } l \in L .
$$

Then for $M, N$ right $A$-modules $a \in A, f \in \operatorname{Hom}(M, N),\left[f, a^{r}\right] \in \operatorname{Hom}$ $(M, N)$ is defined by

$$
\left[f, a^{r}\right](\dot{m})=f(m a)-f(m) a, \text { for } m \in M .
$$

The most direct definition of right differential operators follows the definition above [3, (2.1.1), p. 210]. 
DEFINITION 1.1.

$$
\begin{aligned}
\mathscr{D}_{A}^{0}(M, N) & =\left\{f \in \operatorname{Hom}(M, N) \mid\left[f, a^{r}\right]=0 \text { for all } a \in A\right\} \\
& =\operatorname{Hom}_{A}(M, N),
\end{aligned}
$$

and inductively for $1 \leqq n \in Z$,

$$
\mathscr{D}_{A}^{n}(M, N)=\left\{f \in \operatorname{Hom}(M, N)\left[\left[f, a^{r}\right] \in \mathscr{D}_{A}^{n-1}(M, N) \text { for all } a \in A\right\} .\right.
$$

$\mathscr{D}_{A}(M, N)$ denotes $\cup \mathscr{D}_{A}^{n}(M, N)$. The elements of $\mathscr{D}_{A}(M, N)$ are called right differential operators from $M$ to $N$. The elements of $\mathscr{D}_{A}^{n}(M, N)$ are called $n$th order right differential operators from $M$ to $N$.

Note that if $\mathscr{D}_{A}^{-1}(M, N) \equiv\{0\}$ then

$$
\mathscr{D}_{A}^{0}(M, N)=\left\{f \in \operatorname{Hom}(M, N) \mid\left[f, a^{r}\right] \in \mathscr{D}_{A}^{-1}(M, N) \text { for all } a \in A\right\} \text {. }
$$

Right derivations-used extensively in sections five and six-are first order differential operators. If $M=A$ and $d: A \rightarrow N$ satisfies $d(\alpha \alpha)=d(a) \alpha+d(\alpha) \alpha$ for all $a, \alpha \in A$ then $d$ is called a right derivation. It is easily checked that $\left[d, \alpha^{r}\right](\alpha)=d(\alpha) a$ so that $\left[d, \alpha^{r}\right]=$ $d(\alpha) \in N=\operatorname{Hom}_{A}(A, N)=\mathscr{D}_{A}^{0}(A, N)$ and $d \in \mathscr{D}_{A}^{1}(A, N)$.

For $a$ in the center of $A, a^{r} \in \operatorname{Hom}_{A}(L, L)=\mathscr{D}_{A}^{0}(L, L)$.

The assertion in [3, $(2.1 .1, b)$, p. 210] that "the associated graded algebra is commutative" is not correct. For example if $A$ is a field and $M$ a vector space of dimenston at least 2 then $\mathscr{D}_{A}^{\circ}(M, M)=\operatorname{End}_{A} M$ which is not commutative. What is correct is that the elements of positive degree in the associated graded algebra are central; i.e., all the noncommutativity occurs in degree zero. This correction and all the rest of [3, (2.1.1)] goes over to the case of $A$ noncommutative:

Lemma 1.2. (a) Let $M, N, P$ be right $A$-modules. The composition map

$$
\begin{aligned}
\operatorname{Hom}(M, N) \times \operatorname{Hom}(N, P) & \longrightarrow \operatorname{Hom}(M, P) \\
(u, v) & \longrightarrow v \circ u
\end{aligned}
$$

carries $\mathscr{D}_{A}^{r}(M, N) \times \mathscr{D}_{A}^{s}(N, P)$ into $\mathscr{D}_{A}^{r+s}(M, P)$. If $u \in \operatorname{Hom}_{A}(M, N)$ and $v \in \operatorname{Hom}_{A}(N, P)$ then

$$
v \circ \mathscr{D}_{A}^{n}(M, N) \subset \mathscr{D}_{A}^{n}(M, P) \supset \mathscr{D}_{A}^{r}(N, P) \circ u .
$$

(b) Under composition of endomorphisms $\left\{\mathscr{D}_{A}{ }^{n}(M, M)\right\}$ is a filtered subalgebra of End $M$. If $u \in \mathscr{D}_{A}^{r}(M, M), v \in \mathscr{D}_{A}^{s}(M, M)$ then $[u, v]=u \circ v-v \circ u \in \mathscr{D}_{A}^{r+s-1}(M, M)$ when $0 \leqq r, s \in Z$ and $r+s \geqq 1$, so that $u \circ v \equiv v \circ u \bmod \mathscr{D}_{A}^{r+s-1}(M, M)$ and in the associated graded algebra of $\left\{\mathscr{D}_{A}^{n}(M, M)\right\}$ the center is 


\section{$Z \mathscr{D}_{A}^{0}(M, M) \oplus \bigoplus_{i=0}^{\infty}\left(\mathscr{D}_{A}^{i+1}(M, M) / \mathscr{D}_{A}^{i}(M, M)\right)$}

where $Z \mathscr{D}_{A}^{\circ}(M, M)$ is the center of $\mathscr{D}_{A}^{\circ}(M, M)$.

(c) Let $A$ and $B$ be algebras and let $M, N$ be right $A$-modules and $M^{\prime}, N^{\prime}$ be right $B$-modules (so $M \otimes M^{\prime}$ and $N \otimes N^{\prime}$ are right $A \otimes B$-modules). Then natural map

$$
\operatorname{Hom}(M, N) \otimes \operatorname{Hom}\left(M^{\prime}, N^{\prime}\right) \longrightarrow \operatorname{Hom}\left(M \otimes M^{\prime}, N \otimes N^{\prime}\right),
$$

carries

$\mathscr{D}_{A}^{r}(M, N) \otimes \mathscr{D}_{B}^{s}\left(M^{\prime}, N^{\prime}\right) \quad$ into $\quad \mathscr{D}_{A \otimes B}^{r+s}\left(M \otimes M^{\prime}, N \otimes N^{\prime}\right)$.

Proof. (a) If $a \in A, u \in \operatorname{Hom}(M, N), v \in \operatorname{Hom}(N, P)$, the first part of (a) follows by induction on $r+s$ using the identity:

$$
\left[v \circ u, a^{r}\right]=\left[v, a^{r}\right] \circ u+v \circ\left[u, a^{r}\right] \in \operatorname{Hom}(M, P) .
$$

The second part of (a) then follows from the first since $\operatorname{Hom}_{A}(M, N)=\mathscr{D}_{A}^{\circ}(M, N)$ and $\operatorname{Hom}_{A}(N, P)=\mathscr{D}_{A}^{0}(N, P)$.

(b) The first part of (b) follows from (a) taking $M=N=P$. In particular $\mathscr{D}_{A}^{0}(M, M)$ is a subalgebra of $\mathscr{D}_{A}(M, M)$ so that for $u, v \in \mathscr{D}_{A}^{\circ}(M, M),[u, v] \in \mathscr{D}_{A}^{\circ}(M, M)$. Using the identity:

$$
\left[[u, v] a^{r}\right]=\left[\left[u, a^{r}\right], v\right]+\left[u,\left[v, a^{r}\right]\right]
$$

for $u, v \in \operatorname{Hom}(M, N)$ the second part of (b) follows by induction on $r+s$ where $r+s \geqq 1$.

(c) Let $u \in \operatorname{Hom}(M, N), v \in \operatorname{Hom}\left(M^{\prime}, N^{\prime}\right), a \in A, b \in B$ then

$$
\left[u \otimes v,(a \otimes b)^{r}\right]=\left(u \circ a^{r}\right) \otimes\left[v, b^{r}\right]+\left[u, a^{r}\right] \otimes\left(b^{r} \circ v\right) .
$$

This identity gives (c) by induction on $r+s$.

For an algebra $A$ let $\bar{A}$ denote the "opposite" algebra where -: $A \stackrel{(a \rightarrow \bar{a})}{\longrightarrow} \bar{A}$ is an algebra anti-isomorphism. $\overline{\bar{A}}$ is identified with $A$ by means of the algebra isomorphism $=: A \stackrel{(a \rightarrow \bar{a})}{\longrightarrow} \overline{\bar{A}}$. As in $[2, \mathrm{p}$. 167] $A^{e}=\bar{A} \otimes A$ is called the "enveloping algebra" of $A$. The two maps

$$
\begin{aligned}
& A \stackrel{e_{0}}{\longrightarrow} A^{e} \stackrel{e_{1}}{\longleftarrow} \bar{A} \\
& a \longrightarrow \overline{1} \otimes \alpha, \bar{a} \otimes 1 \longleftarrow \bar{a}
\end{aligned}
$$

are algebra maps.

Definition 1.7. For an algebra $A$ let $d_{0}$ and $d_{1}$ denote the maps 


$$
\begin{aligned}
& A \stackrel{d_{0}}{\longrightarrow} A^{e} \stackrel{d_{1}}{\longleftarrow} \bar{A} \\
& a \longrightarrow \overline{1} \otimes a-\bar{a} \otimes 1 \longleftarrow \bar{a} .
\end{aligned}
$$

Let $D=\operatorname{Im} d_{0}=\operatorname{Im} d_{1}$ and for $1 \leqq n \in Z, D^{n}$ is the $R$ submodule of $A^{e}$ spanned by monomials of length $n$ of elements of $D$.

For $M, N$ right $A$-modules $\operatorname{Hom}(M, N)$ is a left $A^{e}$-module where for $f \in \operatorname{Hom}(M, N), \bar{a} \otimes \alpha \in A^{e}$

$$
\left\{\begin{array}{l}
(\bar{a} \otimes \alpha) \cdot f=a^{r} \circ f \circ \alpha^{r} \in \operatorname{Hom}(M, N) \text { so } \\
d_{0}(\alpha) \cdot f=\left[f, \alpha^{r}\right] \text { for } \quad a \in A
\end{array}\right.
$$

Unlike the situation with $A$ commutative, $\mathscr{D}_{A}^{n}(M, N)$ - even $\mathscr{D}_{A}^{\circ}(M, N)$ - is not in general an $A^{e}$-submodule of $\operatorname{Hom}(M, N)$; see $[3,(2.2 .1, b)$ p. 212].

$A$ is a left $A^{e}$-module where for $b \in A, \bar{a} \otimes \alpha \in A^{e}$

$$
\left\{\begin{array}{l}
(\bar{a} \otimes \alpha) \cdot b=\alpha b a \quad \text { and } \\
A^{e} \stackrel{\mu}{\longrightarrow} A \\
\bar{a} \otimes \alpha \longrightarrow \alpha a
\end{array}\right.
$$

is a left $A^{e}$-module map.

Clearly $\operatorname{ker} \mu=\left\{x \in A^{e} \mid x \cdot 1_{A}=0\right\}$ a left ideal in $A$.

For $1 \leqq m \in \boldsymbol{Z}$ let $[m]$ denote $\{1,2, \cdots, m\}$; let [0] denote $\varnothing$. For $S$ a finite set ${ }^{\#} S$ denotes the cardinality of $S$. If $\varnothing \neq S \subset[m]$ with $S=\left\{i_{1}<\cdots<i_{l}\right\}$ and $\boldsymbol{a}=a_{1} \times \cdots \times a_{m} \in \overbrace{A \times \cdots \times A}^{m}$ let

$$
\left\{\begin{array}{l}
\boldsymbol{a}_{S}=a_{i_{1}} a_{i_{2}} \cdots a_{i_{l}} \in A \\
{ }_{S} \overline{\boldsymbol{a}}=\bar{a}_{i_{1}} \bar{a}_{i_{2}} \cdots \bar{a}_{i_{l}}=\overline{a_{i_{l}} \cdots a_{i_{2}} a_{i_{1}}} \in \bar{A} \quad \text { and } \\
\boldsymbol{a}_{\varnothing}=1 \in A, \quad \varnothing \bar{a}=\overline{1} \in \bar{A} .
\end{array}\right.
$$

LEMMA 1.11. [3, (2.2.2) p. 213].

(a) $\operatorname{ker} \mu=A^{e} D=e_{0}(A) D=e_{1}(\bar{A}) D$.

(b) $A^{e} D^{m}$ is the left $e_{0}(A)$-submodule or $e_{1}(\bar{A})$-submodule of $A^{e}$ generated by $\left\{d_{0}\left(a_{1}\right) \cdots d_{0}\left(a_{m}\right)\right\}_{a_{1} \times \cdots \times a_{m} \in \overparen{A \times \cdots \times A}}$.

(c) For $\boldsymbol{a}=a_{1} \times \cdots \times a_{m} \in \overbrace{A \times \cdots \times A}^{m}$

$$
d_{0}\left(a_{1}\right) \cdots d_{0}\left(a_{m}\right)=\sum_{S \subset[m]}(-)^{\# S} \overline{\boldsymbol{a}} \otimes \boldsymbol{a}_{[m]-S} \cdot
$$

Proof. Suppose $0=\mu\left(\Sigma \bar{a}_{i} \otimes \alpha_{i}\right)=\Sigma \alpha_{i} a_{i}$. Then 


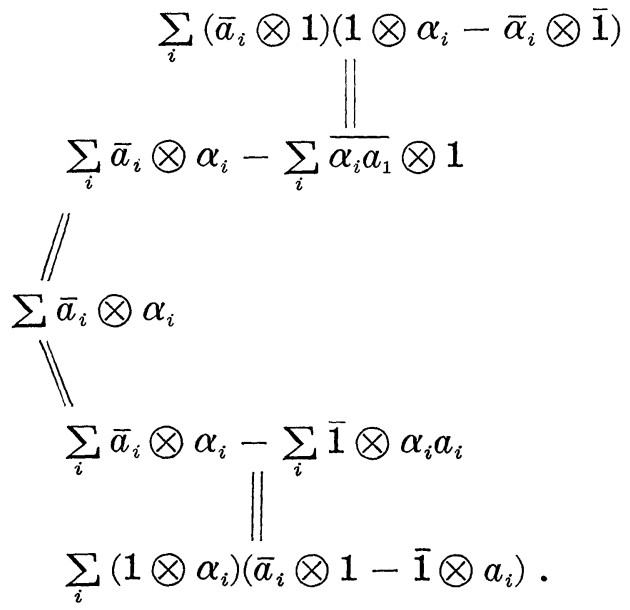

Since $D \subset \operatorname{ker} \mu$ a left ideal this proves (a).

(b) follows from (a).

(c) follows by induction on $m$.

Lemma 1.12. [3, (2.2.3, a, 13$),$ p. 213].

Let $M, N$ be right $A$-modules and $u \in \operatorname{Hom}(M, N)$ for $0 \leqq n \in Z$. The following conditions are equivalent:

(1) $u \in \mathscr{D}_{A}^{n}(M, N)$,

(2) $D \cdot u \in \mathscr{D}_{A}^{n-1}(M, N)$,

(3) $\left(A^{e} D^{n+1}\right) \cdot u=\{0\}$.

Proof. By the inductive definition of $\mathscr{D}_{A}^{n}(M, N)$ and the identity (1.8) $d_{0}(\alpha) \cdot f=\left[f, \alpha^{r}\right],(1)$ and (2) are equivalent. Hence by induction if $u \in \mathscr{D}_{A}^{n}(M, N)$ then $D^{n+1} \cdot u \in \mathscr{D}_{A}^{-1}(M, N)=\{0\}$ and $\left(A^{e} D^{n+1}\right) \cdot u=\{0\}$. Thus (1) and (2) implies (3). Conversely if $\left(A^{e} D^{n+1}\right) \cdot u=\{0\}$, then $D^{n+1} \cdot u=\{0\}$ and $D \cdot\left(D^{n} \cdot u\right)=\{0\}$. Thus by definition $D^{n} \cdot u \subset$ $\mathscr{D}_{A}^{0}(M, N)$. By induction $D^{n-i} \cdot u \subset \mathscr{D}_{A}^{i}(M, N)$ which for $i=n$ given $u \in \mathscr{D}_{A}^{n}(M, N)$.

For an $R$-module $X$ let $\bar{A} \otimes X$ have the left $\bar{A}$-module structure arising from $\bar{A}$. Let $\xi: X \stackrel{(x \rightarrow \overline{1} \otimes x)}{\longrightarrow} \bar{A} \otimes X$. For any left $\bar{A}$-module $Q$ there is the natural bijective correspondence

$$
\left\{\begin{array}{c}
\operatorname{Hom}_{\bar{A}}(\bar{A} \otimes X, Q) \longrightarrow \operatorname{Hom}(X, Q) \\
g \longrightarrow g^{\xi} \\
f^{e} \longleftrightarrow f \\
\text { where for } \bar{a} \otimes x \in \bar{A} \otimes X \\
f^{e}(\bar{a} \otimes x)=\bar{a} f(x) .
\end{array}\right.
$$


A right $A$-module $N$ becomes a left $\bar{A}$-module where $\bar{a} \cdot n \equiv n \cdot a$ for $\bar{a} \in \bar{A}, n \in N$. Suppose $M$ is also a right $A$-module. Then $\bar{A} \otimes M$ is naturally a right $\bar{A} \otimes A=A^{e}$-module. The correspondence $\operatorname{Hom}_{\bar{A}}(\bar{A} \otimes M, N) \leftrightarrow \operatorname{Hom}(M, N)$ ties together a number of module actions by the identity

$$
\begin{aligned}
f^{e}((\bar{b} \otimes m) \cdot(\bar{a} \otimes \alpha)) & =\bar{b} \bar{a} f(m \alpha)=\left(a^{r} \circ f \circ \alpha^{r}\right)^{e}(\bar{b} \otimes m) \\
& =((\bar{a} \otimes \alpha) \cdot f)^{e}(\bar{b} \otimes m)
\end{aligned}
$$

for $f \in \operatorname{Hom}(M, N), \bar{a} \otimes \alpha \in A^{e}, \bar{b} \otimes m \in \bar{A} \otimes M$. The identity (1.8) and (1.4) combine to give

$$
f^{e}\left(z \cdot d_{0}(\alpha)\right)=\left(d_{0}(\alpha) \cdot f\right)^{e}(z)=\left[f, \alpha^{r}\right]^{e}(z)
$$

for $f \in \operatorname{Hom}(M, N), \alpha \in A, z \in \bar{A} \otimes M$.

Definition 1.16. For a right $A$-module $M$ let $J_{n}(M)=$ $\bar{A} \otimes M /\left((\bar{A} \otimes M) \cdot D^{n+1}\right)$. The $\operatorname{map} j_{n}: M \rightarrow J_{n}(M)$ is the composite

$$
M \stackrel{\xi}{\longrightarrow} \bar{A} \otimes M \stackrel{\pi}{\longrightarrow} \bar{A} \otimes M /\left((\bar{A} \otimes M) \cdot D^{n+1}\right)=J_{n}(M) .
$$

$(\bar{A} \otimes M) \cdot D^{n+1}$ is a left $\bar{A}$-submodule of $\bar{A} \otimes M$ and the left $\bar{A}$-module structure on $J_{n}(M)$ is the natural quotient module structure. The left $\bar{A}$-module structure on $J_{n}(M)$ gives $J_{n}(M)$ a right $A$-module structure.

$\left(J_{n}(M), j_{n}\right)$ is the appropriate variation of the " $J$ " defined in $[3$, p. 214] for the noncommutative case.

\section{Universal Property Theorem 1.17 [3, (2.2.6, a) p. 215].}

If $J_{n}(M)$ has the right $A$-module structure arising from its left $\bar{A}$-module structure then $j_{n} \in \mathscr{D}_{A}^{n}\left(M, J_{n}(M)\right)$. If $N$ is any right $A$-module and $u \in \mathscr{D}_{A}^{n}(M, N)$ then there is a unique $g \in \operatorname{Hom}_{-\overline{1}}\left(J_{n}(M)\right.$, $N)=\operatorname{Hom}_{A}\left(J_{n}(M), N\right)$ with $g j_{n}=u$. Thus the map

$$
\begin{aligned}
\operatorname{Hom}_{A}\left(J_{n}(M), N\right)=\operatorname{Hom}_{\bar{A}}\left(J_{n}(M), N\right) & \longrightarrow \mathscr{D}_{A}^{n}(M, N) \\
g & \longrightarrow g j_{n}
\end{aligned}
$$

is bijective.

Proof. If $\bar{A} \otimes M$ has the right $A$-module structure arising from the left $\bar{A}$-module structure and $a, \alpha \in A, \xi \in M$

$$
\begin{aligned}
((\bar{a} \otimes \alpha) \cdot \xi)(m) & =\left(a^{r} \circ \xi \circ \alpha^{r}\right)(m)=\bar{a} \cdot \xi(m \alpha) \\
& =\bar{a} \otimes m \alpha=\xi(m) \cdot(\bar{a} \otimes \alpha) .
\end{aligned}
$$

Thus $\left(D^{n+1} \cdot \xi\right)(M) \subset(\bar{A} \otimes M) \cdot D^{n+1}=\operatorname{ker} \pi$. Since $j_{n}=\pi \xi$ it follows 
$D^{n+1} \cdot j_{n}=0$ and by (1.12) $j_{n} \in \mathscr{D}_{A}{ }^{n}\left(M, J_{n}(M)\right)$.

By (1.2.a) if $g \in \operatorname{Hom}_{\bar{A}}\left(J_{n}(M), N\right)$ then $g j_{n} \in \mathscr{D}_{A}{ }^{n}(M, N)$.

Say $u \in \mathscr{D}_{A}{ }^{n}(M, N)$. By (1.13) $u^{e}$ is the unique element of $\operatorname{Hom}_{\bar{A}}(\bar{A} \otimes M, N)$ with $n^{e} \xi=u$. By (1.15)

$$
u^{e}\left((\bar{A} \otimes M) \cdot D^{n+1}\right)=\left(D^{n+1} \cdot u\right)^{e}(\bar{A} \otimes M)
$$

and $D^{n+1} \cdot u=0$ by (1.12). Hence $u^{e}$ vanishes on ker $\pi$ and there is a unique element $g \in \operatorname{Hom}_{\bar{A}}\left(J_{n}(M), N\right)$ with $g \pi=u^{e}$. Thus $g$ is the unique map with the property:

$$
u=u^{e} \xi=g \pi \xi=g j_{n} .
$$

Let us examine $J_{n}(A)$. This is $A^{e} / A^{e} D^{n+1}$ which has a left $A^{e}$-module structure since $A^{e} D^{n+1}$ is a left ideal. The left $A^{e}$-module structure on $J_{n}(A)$ gives rise to left $A$ and $\bar{A}$-module struct ures from the algebra maps $A \stackrel{e_{0}}{\rightarrow} A^{e} \stackrel{e_{1}}{\leftarrow} \bar{A}$. This left $A$-module structure is the same as in (1.16), (1.17). The left $A$-module structure on $J_{n}(A)$ may be used to give an algernate construction of $J_{n}(M)$ for a right $A$-module $M$. The $j_{n}$ of $J_{n}(A)$ is the composite $A \stackrel{e_{0}}{\rightarrow} A^{e} \stackrel{\pi}{\rightarrow} J_{n}(A)$.

THEOREM 1.18. For a right A-module $M$ there is a unique left $\bar{A}$-module isomorphism $\psi: J_{n}(M) \rightarrow M \otimes_{A} J_{n}(A)$ making the diagram commute!

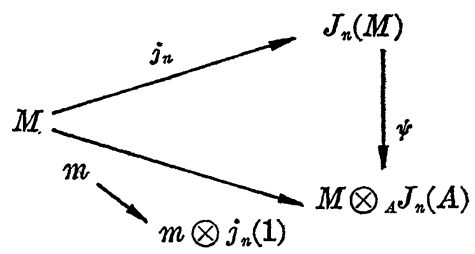

Hence $M \otimes_{A} J_{n}(A)$ together with $M \stackrel{m \rightarrow m \otimes j_{n}(1)}{\longrightarrow} M \otimes_{A} J_{n}(A)$ has the same universal property as $\left(J_{n}(M), j_{n}\right)$ in (1.17).

Proof. Since $A^{e}=\bar{A} \otimes A$ we have

$$
M \otimes_{A} A^{e}=M \otimes_{A}(\bar{A} \otimes A)=\bar{A} \otimes\left(M \otimes_{A} A\right)=\bar{A} \otimes M .
$$

Apply $M \otimes_{A^{-}}$to the exact sequence.

$$
0 \longrightarrow A^{e} D^{n+1} \longrightarrow A^{e} \longrightarrow A^{e} / A^{e} D^{n-1}=J_{n}(A) \longrightarrow 0
$$

to obtain the exact sequence:

$$
M \otimes_{A}\left(A^{e} D^{n+1}\right) \stackrel{\gamma}{\longrightarrow} M \otimes_{A} A^{e} \longrightarrow M \otimes_{A} J_{n}(A) \longrightarrow 0
$$


Using (1.19) to identify $M \otimes_{A} A^{e}$ with $\bar{A} \otimes M$ the image of $\gamma$ is $(\bar{A} \otimes M) \cdot D^{n+1}$. This gives the left $\bar{A}$-module isomorphism

$$
J_{n}(M)=\bar{A} \otimes M /\left((\bar{A} \otimes M) \cdot D^{n+1}\right) \longrightarrow M \otimes_{A} J_{n}(A)
$$

with the commuting diagram.

Recall $\left(1.11\right.$, a) ker $\mu=A^{e} D$. Hence the pair $\left(J_{0}(A), j_{0}\right)$ is naturally equivalent to $(A, I)$ and the left $\bar{A}$-module structure on $A$ arises from the right $A$-module structure $A_{A}$. On occasion $J_{n}(A)$ will assume one of two (opposite) extremal forms. It may happen $A^{e} D^{n+1}=$ $A^{e} D$ for all $0 \leqq n \in Z$. On the other hand there may be a value of $0 \leqq n \in Z$ with $D^{n+1}=\{0\}$. Then $J_{n}(A)=A^{e} / A^{e} D^{n+1}=A^{e}$ and the pair $\left(J_{n}(A), j_{n}\right)$ is simply $\left(A^{e}, e_{0}\right)$.

Definition 1.20. An algebra $A$ is right differentially separable if $A^{e} D=A^{e} D^{2}$. If $D^{n+1}=\{0\}$ then $A$ is called right $n$-differentially stacked.

THEOREM 1.21 .

(a) The following conditions are equivalent:

(i) $A$ is right differentially separable.

(ii) $A^{e} D^{n+1}=A^{e} D$ for all/one $1 \leqq n \in Z$.

(iii) $\left(J_{n}(A), j_{n}\right) \approx(A, I)$ for all/one $1 \leqq n \in Z$.

(iv) $\mathscr{D}_{A}^{n}(M, N)=\mathscr{D}_{A}^{0}(M, N)=\operatorname{Hom}_{A}(M, N)$ for all right $A$-modules $M, N$ and all/one $1 \leqq n \in Z$.

(v) $\mathscr{D}_{A}^{n}(A, N)=\mathscr{D}_{A}^{0}(A, N)=\operatorname{Hom}_{A}(A, N)=N$ for all right $A$-modules $N$ and all/one $1 \leqq n \in Z$.

(b) The following conditions are equivalent:

(i) $A$ is right n-differentially stacked.

(ii) $A^{e} D^{m+1}=\{0\}$ for $n \leqq m \in \boldsymbol{Z}$.

(iii) $\left(J_{n}(A), j_{n}\right) \approx\left(A^{e}, e_{0}\right)$.

(iv) $\left(J_{m}(A), j_{m}\right) \approx\left(A^{e}, e_{0}\right)$ for $n \leqq m \in Z$.

(v) $\mathscr{D}_{A}^{n}(M, N)=\operatorname{Hom}(M, N)$ for all right $A$-modules $M, N$.

(vi) $\mathscr{D}_{A}^{m}(M, N)=\operatorname{Hom}(M, N)$ for all right $A$-modules $M, N$ and $n \leqq m \in \boldsymbol{Z}$.

(vii) $\mathscr{D}_{A}^{n}(A, N)=\operatorname{Hom}(A, N)$ for all right $A$-modules $N$.

(viii) $\mathscr{D}_{A}^{m}(A, N)=\operatorname{Hom}(A, N)$ for all right $A$-modules $N$ and $n \leqq m \in Z$.

Proof. (a) By (1.16) the definition of $J_{n}(A)$ and a remark above (1.20), (ii) and (iii) are equivalent. By (1.18) the universal property of $\left(J_{n}(A), j_{n}\right)$, (iii) and (v) are equivalent. Clearly (iv) implies (v) and by (1.18), (v) implies (iv). Thus (ii), (iii), (iv) and (v) are equivalent. 
Say $A$ is right differentially separable so that $A^{e} D^{2}=A^{e} D$. By induction on $n, A^{e} D^{n+1}, A^{e} D$ for all $1 \leqq n \in Z$. Say $A^{e} D^{n+1}=A^{e} D$ for one $1 \leqq n \in Z$. Then the inclusions $A^{e} D^{n+1} \subset A^{e} D^{2} \subset A^{e} D$ show that $A^{e} D^{2}=A^{e} D$ and $A$ is differentially separable. Thus (i) is equivalent to (ii) and part (a) is proved.

(b) By (1.16), the definition of $J_{n}(A)$ and a remark above (1.20), (i) is equivalent to (iii) and (ii) is equivalent to (iv). By (1.18) the universal property of $\left(J_{i}(A), j_{i}\right)$, (iii) is equivalent to (vii) and (iv) is equivalent to (viii). Clearly (v) implies (vii) and (vi) implies (viii). By (1.18), (vii) implies (v) and (viii) implies (vi). We have established:

$$
\begin{aligned}
& (\mathrm{i}) \Longleftrightarrow \text { (iii) } \Longleftrightarrow \text { (vii) } \Longleftrightarrow(\mathrm{v}) \\
& (\mathrm{ii}) \Longleftrightarrow \text { (iv) } \Longleftrightarrow \text { (viii } \Longleftrightarrow(\mathrm{vi}) \text {. }
\end{aligned}
$$

A is right $n$-differentially stacked if and only if $D^{n+1}=0$ if and only if $A^{e} D^{m+1}=0$ for $n \leqq m \in Z$. Hence (i) and (iii) are equivalent.

So when $A$ is right $n$-differentially stacked $\operatorname{Hom}(M, N)=$ $\mathscr{D}_{A}{ }^{n}(M, N)=\mathscr{D}_{A}(M, N)$ for all right $A$-modules $M, N$. There is a particular test module and test morphism which we now describe.

$A^{e}$ has left $\bar{A}$-module structure from $e_{1}: \bar{A} \rightarrow A^{e}$ which gives $A^{e}$ a right $A$-module structure. (This is not usually the same as the right $A$-module structure arising from $A \stackrel{e_{0}}{\rightarrow} A^{e}$.)

Lemma 1.21. If $e_{0}: A \rightarrow A^{e}$ is a right differential operator of order $n$ with respect to the right A-module structure on $A^{e}$ arising from $e_{1}: \bar{A} \rightarrow A^{e}$ then $A$ is right n-differentially stacked.

Proof. Keeping track that $J_{n}(A)=A^{e} / A^{e} D^{n+1}$ and $j_{n}=\pi e_{0}$ then by (1.17) there is a left $\bar{A}$-module map $f: A^{e} / A^{e} D^{n+1} \rightarrow A^{e}$ making the diagram commute

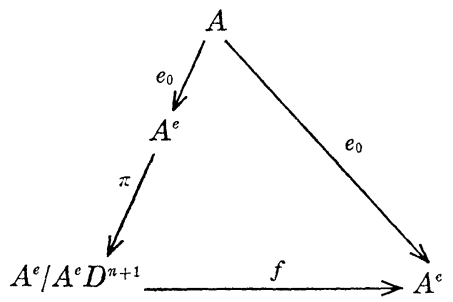

$A^{e}$ is generated by $e_{0}(A)$ as a left $\bar{A}$-module. Hence $f$ is surjective. $\pi f: A^{e} / A^{e} D^{n+1} \rightarrow A^{e} / A^{e} D^{n+1}$ is a left $A$-module map with

$$
(\pi f) j_{n}=(\pi f)\left(\pi e_{0}\right)=\pi\left(f \pi e_{0}\right)=\pi e_{0}=j_{n} .
$$


By the universal property of $\left(J_{n}(A), j_{n}\right)$ it follows that $\pi f$ is the identity map. Since $f$ is surjective $\pi$ must be injective. Hence $\{0\}=\operatorname{ker} \pi=A^{e} D^{n+1} \supset D^{n+1}$.

2. Right differentially stacked algebras. Throughout this section the ground ring $R$ is assumed to be a field. In $[7,1, \mathrm{p}$. $343]$ purely inseparable algebras are defined. An algebra $A$ is purely inseparable if $A^{e} \stackrel{\mu}{\rightarrow} A$ is a projective cover of $A$ as left $A^{e}$-module. By $[7,(13 . b)$ p. 353] a finite dimensional algebra $A$ is purely inseparable if and only if $A / J$ is a purely inseparable field extension of the ground field $R$, where $J$ is the Jacobson radical of $A$.

Pure Inseparability Theorem 2.1. For a finite dimensional algebra $A$ the following conditions are equivalent:

(i) $A$ is a purely inseparable algebra.

(ii) $D$ lies in the Jacobson radical of $A^{e}$.

(iii) $A$ is right n-differentially stacked for some $n$.

Proof. (ii) $\Rightarrow$ (iii). Since $A$ is finite dimensional so is $A^{e}$ and its Jacobson radical to some $(n+1)$ power is zero. Thus by (ii), $D^{n+1}=\{0\}$, and by (1.20) $A$ is right $n$-differentially stacked.

(i) $\Rightarrow$ (ii). We consider characteristic zero and positive characteristic separately. If $R$ has characteristic zero then $A / J$ being a purely inseparable field extension of $R$ implies that $A=R \oplus J$. For $r \in R, y \in J, d_{0}(r+x)=\overline{1} \otimes x+\bar{x} \otimes 1 \in \bar{A} \otimes J+\bar{J} \otimes A$ and $D \subset \bar{A} \otimes J+$ $\bar{J} \otimes A$. Clearly $\bar{A} \otimes J$ and $\bar{J} \otimes A$ (each nilpotent two-sided ideals) lie in the Jacobson radical of $A^{e}$; hence $D$ lies in the Jacobson radical of $A^{e}$. This shows that (i) $\Rightarrow$ (ii) when $R$ has characteristic zero.

Suppose $R$ has positive characteristic $p$. Suppose $K$ is a purely inseparable field extension of $R$ of exponent $s$, i.e., $x^{p^{s}} \in R$ for $x \in K$. If $x_{1}, \cdots, x_{t}$ is a basis for $K$ over $R$ then in $K \otimes K$

$$
\left(1 \otimes x_{i}-x_{i} \otimes 1\right)\left(\left(1 \otimes x_{1}-x_{1} \otimes 1\right)^{p^{s}-1} \cdots\left(1 \otimes x_{t}-x_{t} \otimes 1\right)^{p^{s}-1}\right)
$$

when regrouped will contain

$$
\left(1 \otimes x_{i}-x_{i} \otimes 1\right)^{p^{s}}=1 \otimes x_{i}^{p^{s}}-x_{i}^{p^{s}} \otimes 1=0
$$

since $x_{i}^{p^{s}} \in R$.

$K^{e}=\bar{K} \otimes K$ may be identified with $K \otimes K$ since $K$ is commutative. Since any $x \in K$ is an $R$-linear combination of $\left(x_{i}\right)^{\prime}$ 's it follows that

$$
\{0\}=D^{\left(p^{s}-1\right) t+1} \subset K^{e} .
$$


Since $K^{e}$ is commutative this shows that $K^{e} D$ is a nilpotent ideal and $D$ lies in the Jacobson radical of $K^{e}$.

Let $\gamma: A \rightarrow A / J$ be the natural surjection and let $\underline{\underline{\gamma}}: \bar{A} \rightarrow \bar{A} / \bar{J}$, $\bar{a} \rightarrow \overline{\gamma(a)}$. Then $\bar{\gamma} \otimes \gamma: \bar{A} \otimes A \rightarrow \bar{A} / \bar{J} \otimes A / J$ is a surjective algebra map with kernel $\bar{A} \otimes J+\bar{J} \otimes A$ which lies in the Jacobson radical of $A^{e}$. Since the kernel of $\underline{\bar{\gamma}} \otimes \gamma$ lies in the Jacobson radical of $A^{e}$ it is easily shown that

\section{( $\left.^{*}\right)(\underline{\bar{\gamma}} \otimes \gamma)^{-1}\left(\right.$ Jacobson radical of $\left.(A / J)^{e}\right) \subset$ Jacobson radical of $A^{e}$.}

Since $A / J$ is a purely inseparable field extension of $R$, by what we have shown for $K$ above it follows that $(\bar{\gamma} \times \gamma)(D)$ lies in the Jacobson radical of $(A / J)^{e}$. Thus by $\left(^{*}\right)$ it follows that $D$ lies in the Jacobson radical of $A^{e}$, which completes (i) $\Rightarrow$ (ii).

(iii) $\Rightarrow$ (i). Suppose $A$ is a right $n$-differentially stacked algebra. If $a \in A$ is idempotent then $(\overline{1} \otimes a-\bar{a} \otimes 1)^{3}=(\overline{1} \otimes a-\bar{a} \otimes 1)$ and so

$$
(\overline{1} \otimes a-\bar{a} \otimes 1)=(\overline{1} \otimes a-\bar{a} \otimes 1)^{\text {odd power }} .
$$

Since $D^{n+1}=\{0\}$ it follows

$$
0=(\overline{1} \otimes a-\bar{a} \otimes 1)^{n+1}=(\overline{1} \otimes a-\bar{a} \otimes 1)^{n+2} .
$$

One of these coefficients is odd and so $\overline{1} \otimes a-\bar{a} \otimes 1=0$. Since $R$ is a field $\overline{1} \otimes a-\bar{a} \otimes 1=0$ implies $a \in R$ and hence $a=1$. Thus 1 is the only idempotent of $A$, and $A$ is indecomposable, i.e., $A$ is not of the form $B \oplus C$ where $B$ and $C$ are two-sided ideals in $A$.

Since $A$ is right $n$-differentially stacked $D^{n+1}=\{0\}$ and for $b, a_{0}, \cdots, a_{n} \in A$

$$
d_{0}\left(a_{0}\right) d_{0}\left(a_{1}\right) \cdots d_{0}\left(a_{n}\right)=0
$$

so that

$$
\begin{aligned}
0 & =\left(d_{0}\left(a_{0}\right) \cdots d_{0}\left(a_{n}\right)\right) \cdot b \\
& =\left[\cdots\left[\left[b, a_{n}\right], a_{n-1}\right] \cdots, a_{0}\right]
\end{aligned}
$$

and $A^{-}$is a nilpotent Lie algebra. ( $A^{-}$is $A$ with the Lie structure $[b, a]=b a-a b$.$) Thus by [6,(\mathrm{I}, \mathrm{a}, 7 \Rightarrow 9) \mathrm{p} .103] A / J$ is a field since $A$ is indecomposable. Let $\gamma: A \rightarrow A / J$ be the natural algebra map. Let $D_{A}$ be the $D \subset A^{e}$ for $A$ and $D_{A / J}$ be the $D \subset(A / J)^{e}$ for $A / J$. Since $(\bar{\gamma} \otimes \gamma)\left(D_{A}\right)=D_{A / J}$ if $D_{A}^{n+1}=\{0\}$ then $(\bar{\gamma} \otimes \gamma)\left(D_{A}^{n+1}\right)=$ $D_{A \mid J}^{n+1}=\{0\}$ and $A / J$ is right $n$-differentially stacked.

We are reduced to showing that if $A$ is a field which is right $n$-differentially stacked then $A$ is a purely inseparable field extension. Let $S$ be the unique maximal separable extension of $R$ in $A$. Since 
$R$ is a field the natural map $S^{e} \rightarrow A^{e}$ is injective. $D_{S}$ maps into $D_{A}$; hence, $D_{S}^{n+1}=\{0\}$. However, since $S$ is a separable field extension by [2, (7.10), p. 179] and [2, (7.9), p. 179], $S^{e}$ is semi-simple. In particular $\operatorname{ker} \mu=S^{e} D_{S}$ is an idempotent ideal if not zero. Thus $D_{S}^{n+1}=\{0\}$ implies $D_{S}=\{0\}$. If $s \in S-R$ then $\overline{1} \otimes s-\bar{s} \otimes 1 \neq 0$ since $R$ is a field. Hence $S=R$ and $A$ is a purely inseparable field extension of $S=R$.

3. Algebra maps. The proof of the pure inseparability theorem suggests several techniques which have further application. These technique are presented here.

Throughout this section $A$ and $B$ are $R$-algebras and $\gamma: A \rightarrow B$ an algebra map. The algebra map $\bar{A} \stackrel{\bar{a} \rightarrow \overline{r(a)}}{\longrightarrow} \bar{B}$ is denoted $\bar{\gamma} . \quad D_{A}$ denotes the $R$-submodule $\operatorname{Im}\left(A \stackrel{d_{0}}{\rightarrow} A^{e}\right)$ and $D_{B}$ denotes the $R$-submodule $\operatorname{Im}\left(B \stackrel{d_{0}}{\rightarrow} B^{e}\right)$. Let $J_{n}^{A}$ denote $A^{e} / D_{A}^{n+1}$ and $J_{n}^{B}$ denote $B^{e} / D_{B}^{n+1}$.

Let $\gamma^{e}$ denote the algebra map $\bar{\gamma} \otimes \gamma: A^{e} \rightarrow B^{e}$. Clearly $\gamma\left(D_{A}\right) \subset$ $D_{B}$ so that $\gamma^{e}\left(A^{e} D_{A}^{n+1}\right) B \subset D_{B}^{n+1}$ and $\gamma^{e}$ induces the unique map $\gamma^{J}$ making the diagram commute:

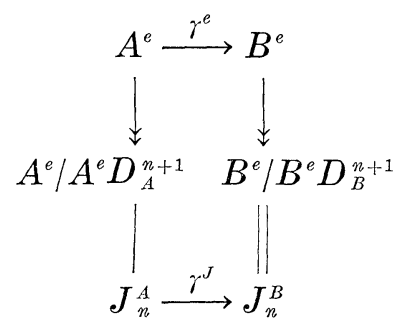

If $X$ is a right [left] module for $B(\bar{B})\left(\left(B^{e}\right)\right)$ it become svia $\gamma(\bar{\gamma})$ $\left(\left(\bar{\gamma}^{e}\right)\right)$ a right [left] module for $A(\bar{A})\left(\left(A^{e}\right)\right)$. The map $\gamma^{J}$ is a left $A^{e}$-module map.

If $M_{B}$ and $N_{B}$ are right $B$-modules and $f \in \mathscr{D}_{B}^{n}(M, N)$ then $f$ is a differential operator with respect to $A$. I.e., $f \in \mathscr{D}_{A}^{n}(M, N)$. In particular $j_{n}^{B} \in \mathscr{D}_{A}^{n}\left(B, J_{n}^{B}\right)$ so that $j_{n}^{B} \gamma \in \mathscr{D}_{A}{ }^{n}\left(A, J_{n}^{B}\right) . \gamma^{J} \in \operatorname{Hom}_{\bar{A}}\left(J_{n}^{A}, J_{n}^{B}\right)$ is the unique map with the property $\gamma^{J} j_{n}^{A}=j_{n}^{B} \gamma$.

Theorem 3.2. (a) If $A$ and $B$ are flat $R$-modules and $\gamma$ is injective then $\gamma^{e}$ is injective.

(b) If $\gamma^{e}$ is injective and $B$ is right $n$-differentially stacked then so is $A$.

(c) Suppose $\gamma$ is surjective:

(i ) $\gamma^{J}: J_{n}^{A} \rightarrow J_{n}^{B}$ is surjective.

(ii) If $A$ is right differentially separable so is $B$.

(iii) If $A$ is right n-differentially stacked so is $B$. 
(iv) If $(\operatorname{ker} \gamma)^{t}=\{0\}$ and $B$ is right n-differentially stacked then $A$ is right $(2 n t+2 t-n-2)$-differentially stacked.

Proof. (a) If $\gamma$ is injective so is $\bar{\gamma}$. If $A$ is a flat $R$-module so is $\bar{A}$ since $A \stackrel{(a \rightarrow \bar{a})}{\longrightarrow} \bar{A}$ is an $R$-module isomorphism. $\gamma^{e}$ factors

$$
\bar{A} \otimes A \stackrel{I \otimes r}{\longrightarrow} \bar{A} \otimes B \stackrel{\bar{r} \otimes I}{\longrightarrow} \bar{B} \otimes B
$$

giving part (a).

(b) Since $\gamma^{e}\left(D_{A}\right) \subset D_{B}$ and $\gamma^{e}$ is an injective algebra map the condition $D_{B}^{n+1}=\{0\}$ implies $D_{A}^{n+1}=\{0\}$.

(c) If $\gamma$ is surjective so is $\underline{\bar{\gamma}}$ and $\underline{\bar{q}} \otimes \gamma=\gamma^{e}$, which implies $\gamma^{J}$ is surjective as claimed in (i). Also $\gamma^{e}\left(D_{A}\right)=D_{B}$. Thus if $A^{e} D_{A}=$ $A^{e} D_{A}^{2}$ applying $\gamma^{e}$ gives

$$
\begin{aligned}
B^{e} D_{B} & =\gamma^{e}\left(A^{e}\right) \gamma^{e}\left(D_{A}\right)=\gamma^{e}\left(A^{e} D_{A}\right)=\gamma^{e}\left(A^{e} D_{A}^{2}\right) \\
& =\gamma^{e}\left(A^{e}\right) \gamma^{e}\left(D_{A}\right)^{2}=B^{e} D_{B}^{2}
\end{aligned}
$$

which proves (ii).

If $D_{A}^{n+1}=\{0\}$ applying $\gamma^{e}$ gives $D_{B}^{n+1}=\gamma^{e}\left(D_{A}\right)^{n+1}=\gamma^{e}\left(D_{A}^{n+1}\right)=\{0\}$ which proves (iii).

(iv) Let $J=\operatorname{Ker} \gamma \subset A . \quad \bar{J} \subset \bar{A}$ is $\operatorname{Ker} \bar{\gamma}$ and by right exactness of $\otimes$ the kernel of $\gamma^{e}$ is the image of $\bar{A} \otimes J+\bar{J} \otimes A$ in $\bar{A} \otimes A=A^{e}$. If $J^{t}=\{0\}$ then $\left(\operatorname{Ker} \gamma^{e}\right)^{2 t-1}=\{0\}$. If $D_{B}^{n+1}=\{0\}$ then $\{0\}=\gamma^{e}\left(D_{A}\right)^{n+1}=$ $\gamma^{e}\left(D_{A}^{n+1}\right)$ and $D_{A}^{n+1} \subset \operatorname{Ker} \gamma^{e}$. Thus

$$
\{0\}=\left(D_{A}^{n+1}\right)^{2 t-1}=D_{A}^{(2 n t+2 t-n-2)+1}
$$

and $A$ is $(2 n t+2 t-n-2)$ - differentially stacked, proving (iii).

4. Base extension. Throughout this section $S$ is a commutative $R$-algebra. If $A$ is an $R$-algebra, $S \otimes A$ is an $S$-algebra and an $R$-algebra. Thus $J_{n}(S \otimes A)$ as an $R$-algebra or as an $S$-algebra both make sense and are different in general. $(S \otimes A)^{e}$ as an $R$-algebra or $S$-algebra make sense and are different in general. Any $S$-algebra $\mathscr{C}$ is naturally an $R$-algebra and $\mathscr{A}^{e}$ has ambigious meaning. To avoid confusion we shall tack an $S$ onto symbols (functors) when working with $S$-algebras and there will be no $S$ when working as $R$-algebras. Thus

$$
D_{\mathscr{A}}^{S} \subset \mathscr{A}^{e_{S}}=\mathscr{A} \otimes_{S} \mathscr{A}
$$

while

$$
D_{\mathscr{A}} \subset \mathscr{A}^{e}=\mathscr{A} \otimes \mathscr{A} .
$$

Suppose $A$ is an $R$-algebra $M, N$ right $A$-modules and $X$ an 
$R$-module. $\quad X \otimes N$ has the right $A$-module structure arising from $N_{A}$. The natural map

$$
\begin{aligned}
X \otimes \operatorname{Hom}_{\bar{A}}\left(J_{n}(M), N\right) & \longrightarrow \operatorname{Hom}_{\bar{A}}\left(J_{n}(M), X \otimes N\right) \\
x \otimes f & \longrightarrow(z \longrightarrow x \otimes f(z))
\end{aligned}
$$

is defined in $[1, \S 9,(8), \mathrm{p} .37]$. The map is bijective when $X$ is a finitely generated projective $R$-module. (4.1) becomes

$$
\begin{aligned}
X \otimes \mathscr{D}_{A}{ }^{n}(M, N) & \longrightarrow \mathscr{D}_{A}{ }^{n}(M, X \otimes N) \\
x \otimes f & \longrightarrow(m \longmapsto x \otimes f(m))
\end{aligned}
$$

which is an isomorphism if $X$ is a finitely generated projective $R$-module.

There is a natural isomorphism $S \otimes A^{e} \stackrel{\simeq}{\longrightarrow}(S \otimes A)^{e} S$ where

$$
s \otimes \bar{a} \otimes \alpha \longrightarrow \overline{(s \otimes a)} \otimes_{S}(1 \otimes \alpha)=\overline{(1 \otimes a)} \otimes \otimes_{S}(s \otimes \alpha) .
$$

We apply $S \otimes$ - to the exact sequence

$$
0 \longrightarrow A^{e} D^{n+1} \longrightarrow A^{e} \longrightarrow A^{e} / A^{e} D^{n+1}=J_{n}(A) \longrightarrow 0
$$

to get

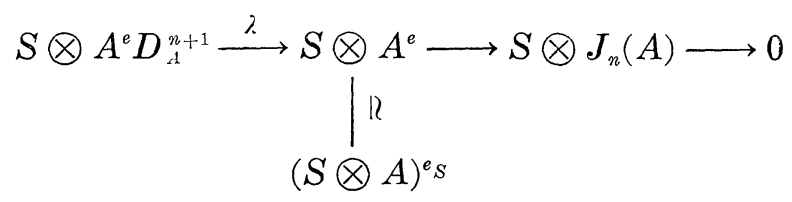

The image of $\lambda$ in $S \otimes A^{e}$ corresponds to the image of $\lambda^{\prime}$ in $(S \otimes A)^{e} S$ for

$$
0 \longrightarrow(S \otimes A)^{e} S D_{S \otimes: A, S}^{n+1} \stackrel{\lambda^{\prime}}{\longrightarrow}(S \otimes A)^{e} S \longrightarrow J_{n}^{S}(S \otimes A) \longrightarrow 0
$$

Thus we have the commutative diagram with exact rows

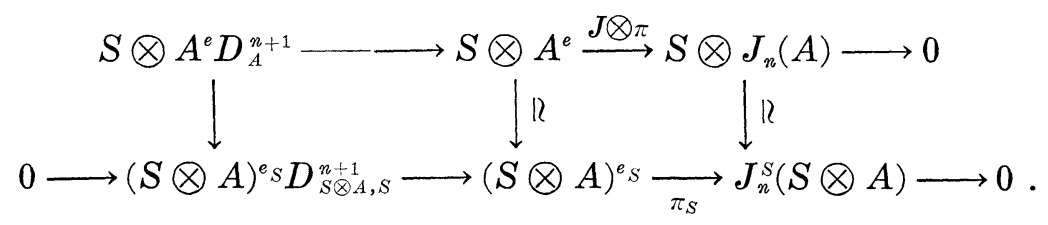

The right hand isomorphism in (4.3) fits into the commutative diagram

$$
S \otimes J_{n}(A) \stackrel{\simeq}{\simeq} J_{n}^{S}(S \otimes A)
$$


Base Change Lemma 4.5. (a) If $M$ is a right A-module and $N$ a right $S \otimes A$-module the correspondence

$$
\begin{aligned}
\mathscr{D}_{S \otimes A}^{n}, S & (S \otimes M, N) \\
f & \longrightarrow\left(m \longmapsto \mathscr{D}_{A}^{n}(M, N)\right. \\
& \longrightarrow f(1 \otimes m))
\end{aligned}
$$

is bijective with inverse

$$
\begin{aligned}
\mathscr{D}_{A}^{n}(M, N) & \longrightarrow \mathscr{D}_{S \otimes A, S}^{n}(S \otimes M, N) \\
g & \longrightarrow(s \otimes m \longmapsto g(m) \cdot(s \otimes 1)) .
\end{aligned}
$$

(b) $\left(J_{n}^{S}(S \otimes A), j_{n, S}\right) \approx\left(S \otimes J_{n}(A), I \otimes j_{n}\right)$.

(c) If $A$ is right differentially separable then $S \otimes A$ is a right differentially separable S-algebra. If $S$ is faithfully flat $R$-module and $S \otimes A$ is a right differentially separable S-algebra then $A$ is right differentially separable.

(d) If $A$ is right $n$-differentially stacked then $S \otimes A$ is right n-differentially stacked as an S-algebra. If $S$ is a faithfully flat $R$-module and $S \otimes A$ is right $n$-differentially stacked as an $S$ algebra then $A$ is right n-differentially stacked.

(e) If $M, N$ are right $A$-modules there is a natural map

$$
\begin{aligned}
S \otimes \mathscr{D}_{A}^{n}(M, N) & \longrightarrow \mathscr{D}_{S \otimes A, S}^{n}(S \otimes M, S \otimes N) \\
s_{1} \otimes f & \longrightarrow\left(s_{2} \otimes m \longmapsto s_{1} s_{2} \otimes f(m)\right)
\end{aligned}
$$

which is bijective if $S$ is a finitely generated projective $R$-module.

Proof. (a) may be verified directly or follows from (b) and (1.18).

(b) is simply (4.4).

(c) $A$ is right differentially separable if and only if the natural map

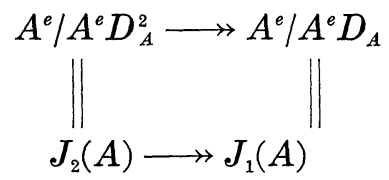

is an isomorphism by (1.20). Using (4.3) we have the commutative diagram

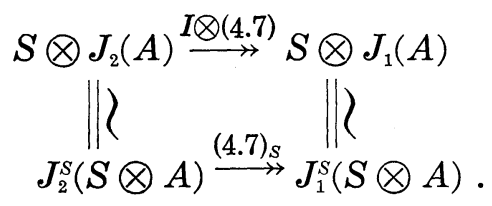

Thus if (4.7) is an isomorphism so is $(4.7)_{S}$ and $S \otimes A$ is a right 
differentially separable $S$-algebra. Conversely if $(4.7)_{S}$ is an isomorphism and $S$ is a faithfully flat $R$-module then from (4.8) it follows that (4.7) is an isomorphism and $A$ is right differentially separable.

(d) $A$ is right $n$-differentially stacked if and only if the natural map

$$
A^{e} \stackrel{\pi}{\longrightarrow} A^{e} / A^{e} D_{A}^{n+1}=J_{n}(A)
$$

is an isomorphism by (1.20). Using (4.3) we have the commutative diagram

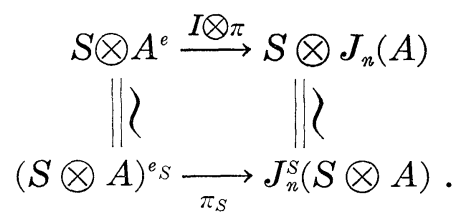

Thus if $\pi$ is an isomorphism so is $\pi_{S}$ and $S \otimes A$ is right $n$-differentially stacked as an $S$-algebra. Conversely if $\pi_{S}$ is an isomorphism and $S$ is a faithfully flat $R$-module then from (4.9) $\pi$ must be an isomorphism and $A$ is right $n$-differentially stacked.

(e) The given map factors

$$
S \otimes \mathscr{D}_{A}^{n}(M, N) \stackrel{(4.2)}{\longrightarrow} \mathscr{D}_{A}^{n}(M, S \otimes N) \stackrel{(a)}{\longrightarrow} \mathscr{D}_{S \otimes A, S}^{n}(S \otimes M, S \otimes N)
$$

where the map (a) is given to part (a) of this lemma using $S \otimes N$ for $N$ in part (a). The map (a) is bijective and (4.2) is bijective when $X=S$ is a finitely generated projective $R$-module.

\section{The right derivative.}

Definition 5.1. For an algebra $A$ and right $A$-module $M$, an $R$-module map $f: A \rightarrow M$ is a right derivation if $f(a b)=f(a) \cdot b+f(b) \cdot a$ for all $a, b \in A$. $R \operatorname{der}(A, M)$ denotes the ( $R$-module of) right derivations from $A$ to $M$.

An interesting example of right derivation arises from skew commutative graded algebras such as the exterior algebra. For such an algebra projection onto the odd graded part is (readily verified to be) a right derivation.

If $N$ is an $A$-bimodule and $g: A \rightarrow N$ is an $R$-module map satisfying $g(a b)=a g(b)+g(a) b$ for all $a, b \in A$ then $g$ is called a derivation as usual. $\operatorname{Der}(A, M)$ denotes the $R$-module of derivations from $A$ to $N$.

Later in the section (5.4), (5.7) we show how right derivations give rise to derivations by a change of module. The derivations 
which arise in this fashion are inner, however. In the commutative theory derivations arise as the first order differential operators vanishing on 1 and they are represented by the Kähler module. This all goes through for right derivations in the noncommutative theory as we show in (5.11), (5.12).

If $f: A \rightarrow M$ is a right derivation and $h: M \rightarrow N$ a map of right $A$-modules then $h f: A \rightarrow N$ is a right derivation. This fact is used frequently without special mention.

$\operatorname{Hom}(A, M)$ has the $A$-bimodule structure arising from $A_{A}$ and $M_{A}$. Specifically for $a, \alpha, b \in A, f \in \operatorname{Hom}(A, M)$

$$
(a \cdot f \cdot \alpha)(b) \equiv f(b a) \alpha .
$$

For $m \in M$ and $h \in \operatorname{Hom}(A, M)$ let $m^{l} \in \operatorname{Hom}(A, M)$ and $h^{l}, \delta h \in$ $\operatorname{Hom}(A, \operatorname{Hom}(A, M))$ be defined by

$$
\left.\begin{array}{l}
m^{l}(a)=m a \in M \\
h^{l}(a)=(h(a))^{l} \in \operatorname{Hom}(A, M) \\
\delta h(a)=a \cdot h-h \cdot a \in \operatorname{Hom}(A, M)
\end{array}\right\} \text { for } a \in A .
$$

Note that under $M \stackrel{l}{\rightarrow} \operatorname{Hom}(A, M) \quad M$ maps isomorphically to $\operatorname{Hom}_{A}(A, M)$ and this gives the usual identification between $M$ and $\operatorname{Hom}_{A}(A, M) . \quad M^{l}$ denotes the image of $l$ in $\operatorname{Hom}(A, M)$.

Proposition 5.4. (a) For $g \in \operatorname{Hom}(A, M)$ the following conditions are equivalent:

(i) $g$ is a right derivation

(ii) $\delta g=g^{l}$

(iii) $\delta g=h^{l}$ for some $h \in \operatorname{Hom}(A, M)$ and $g(1)=0$

(iv) $g^{l}: A \rightarrow \operatorname{Hom}(A, M)$ is a derivation

(v) $g \in \mathscr{D}_{A}{ }^{1}(A, M)$ and $g(1)=0$

(vi) for $a_{1}, \cdots, a_{n} \in A$

$$
g\left(a_{1} a_{2} \cdots a_{n}\right)=\sum_{i=1}^{n} g\left(a_{i}\right) a_{1} \cdots \widehat{a_{i}} \cdots a_{n} .
$$

(b) If $g: A \rightarrow M$ is a right derivation the following conditions are satisfied:

(i) for $a, b \in A, g(a b)=g(b a)$

(ii) for $a_{1}, \cdots, a_{n}, c \in A$

$$
\sum_{i=1}^{n} g\left(a_{i}\right) \cdot\left[\left(a_{1} a_{2} \cdots \widehat{a_{i}} \cdots a_{n}\right), c\right]=0
$$

(iii) for invertible $a \in A$,

$$
g\left(a^{-1}\right)=-g(a) \cdot a^{-2}
$$


(iv) for $a \in A$ and $0 \leqq n \in Z$, (all $n \in Z$ if $a$ is invertible),

$$
g\left(a^{n}\right)=n g(a) \cdot a^{n-1}
$$

(v) for an idempotent $e \in A, g(e)=0$,

(vi) Ker $g$ is a subalgebra of $A$ with the property that if an element of $\operatorname{Ker} g$ is invertible in $A$ the inverse lies in Ker $g$.

(vii) $g$ is (Ker $g$ )-linear, i.e., for $a \in \operatorname{Ker} g, b \in A$

$$
g(b a)=g(b) \cdot a \text {. }
$$

(c) $M^{l} \subset \mathscr{D}_{A}{ }^{1}(A, M) \supset R \operatorname{der}(A, M)$ and $\mathscr{D}_{A}{ }^{1}(A, M)$ is the direct sum of $M^{l}$ and $R \operatorname{der}(A, M)$ as follows:

$$
\begin{gathered}
\mathscr{D}_{A}^{1}(A, M)=M^{l} \oplus R \operatorname{der}(A, M) \\
\left.\in \quad \in \quad \in \quad \in \quad(1)^{l}\right) .
\end{gathered}
$$

Proof. (i ) $\Rightarrow$ (ii). For $a, b \in A \delta g(a)$ applied to $b$ gives

$$
\begin{aligned}
(a \cdot g-g \cdot a)(b) & =g(a b)-g(b) a \\
& =g(a) b+g(b) a-g(b) a=g(a) b
\end{aligned}
$$

which is $g^{l}(a)$ applied to $b$.

(ii) $\Rightarrow$ (iii). $\quad h$ may be chosen as $g$. Furthermore by (ii) $g^{l}(1)=$ $\delta g(1)=0$. And $g^{l}(1)=0$ implies $g(1)=0$ since $g(1)$ is $g^{l}(1)$ applied to 1 .

(ii) $\Rightarrow$ (iv), since $g^{l}=\delta g$ an inner derivation.

(iv) $\Rightarrow$ (i). If $g^{l}$ is a derivation then for $a, b \in A, g^{l}(a b)=$ $a g^{l}(b)+g^{l}(a) b \in \operatorname{Hom}(A, M)$. Applying both sides of the equation to $1 \in A$ gives $g(a b) \cdot 1=g(b) \cdot(1 a)+(g(a) \cdot 1) \cdot b$. Thus $g$ is a right derivation.

(iii) $\Rightarrow(\mathrm{v})$. For $a \in A$

$$
\delta g(a)=\left[g, a^{r}\right]
$$

where $\left[g, a^{r}\right]$ is defined above (1.1). Hence by (iii) for all $a \in A$, $\left[g, a^{r}\right]=g^{l}(a)=g(a)^{l} \in \operatorname{Hom}_{A}(A, M)=\mathscr{D}_{A}^{0}(A, M)$. Thus by Definition (1.1) $g \in \mathscr{D}_{A}^{1}(A, M)$.

(v) $\Rightarrow(\mathrm{i})$. By (v) and (1.1) for $a, b \in A \quad 0=\left[\left[g, b^{r}\right], a^{r}\right] . \quad$ Apply this to $1 \in A$ and expand to get

$$
0=g(a b)-g(a) b-g(b) a+g(1) b a .
$$

Since $g(1)=0$ it follows $g(a b)=g(a) b+g(b) a$ and $g \in R \operatorname{der}(A, M)$.

(vi) $\Rightarrow$ (i) is clear letting $n=2$.

(i) $\Rightarrow$ (vi) goes by induction on $n$. The result is true be definition of right derivation for $n=2$. Say the result is true for $n-1$. 
Write $a_{1} \cdots a_{n}$ as $\left(a_{1} \cdots a_{n-1}\right) a_{n}$ and use the right derivation property and inductive hypothesis to get the case $n$.

$(b, i)$. Both $g(a b)$ and $g(b a)$ equal $g(a) b+g(b) a$ since addition is commutative.

(ii). By (a, vi)

$$
g\left(c a_{1} \cdots a_{n}\right)=g(c) a_{1} \cdots a_{n}+\sum_{i=1} g\left(a_{i}\right) c a_{1} \cdots \widehat{a}_{i} \cdots a_{n} .
$$

Using the right derivation property and (a, vi) again

*

$$
\begin{aligned}
g\left(c\left(a_{1} \cdots a_{n}\right)\right)=g(c) a_{1} \cdots & a_{n}+g\left(a_{1} \cdots a_{n}\right) c \\
\| & \widehat{N}\left(a_{i}\right) a_{1} \cdots \widehat{a_{i}} \cdots a_{n} c .
\end{aligned}
$$

Equating $(*)$ and $(\stackrel{*}{*})$ and cancelling the $g(c) a_{1} \cdots a_{n}$ terms gives

$$
\sum_{i=1}^{n} g\left(a_{i}\right) c a_{1} \cdots \widehat{a_{i}} \cdots a_{n}=\sum_{i=1}^{n} g\left(a_{i}\right) a_{1} \cdots \widehat{a_{i}} \cdots a_{n} c
$$

which is the same as (b, ii).

(b, iii). For $a, a^{-1} \in A$

$$
0=g(1)=g\left(a a^{-1}\right)=g(a) a^{-1}+g\left(a^{-1}\right) a
$$

which gives the desired formula.

(b, iv). For $1 \leqq n \in Z$ the result follows from (a, vi) with $a=$ $a_{1}=a_{2}=\cdots=a_{n}$. If $a$ is invertible and $-2 \geqq n \in Z$ write $a^{n}=$ $\left(a^{-1}\right)^{-n}$ and use what we have shown so far (since $2 \leqq-n \in Z$ ) together with (b, iii).

$(\mathrm{b}, \mathrm{v})$. Since $e$ is idempotent

$$
\begin{gathered}
g(e) \quad g\left(e^{2}\right) \quad g\left(e^{3}\right) \\
\| \\
2 g(e) \cdot e \quad 3 g(e) \cdot e^{2}=3 g(e) \cdot e \\
g(e) \cdot e=0 \text { since } 2 g(e) \cdot e=3 g(e) \cdot e . \\
g(e)=0 \text { since } g(e)=2 g(e) \cdot e .
\end{gathered}
$$

(b, vi). Being idempotent $1 \in \operatorname{Ker} g$. For $u, v \in \operatorname{Ker} g$

$$
g(u v)=g(u) v+g(v) u=0 .
$$

Hence Ker $g$ is a subalgebra of $A$. The assertion about inverses follows from ( $b$, iii).

(b, vii). If $a \in \operatorname{Ker} g, b \in A$

$$
g(b a)=g(b) a+\underbrace{g(a) b}_{0}=g(b) a .
$$


(c) Clearly $M^{l} \subset \mathscr{D}_{A}^{1}(A, M) \supset R \operatorname{der}(A, M)$ and the sum is direct. If $h \in \mathscr{D}_{A}^{1}(A, M)$ then $h-h^{l}(1) \in \mathscr{D}_{A}^{1}(A, M)$ and vanishes on 1 . Hence by $(a, v), h-h^{l}(1) \in R \operatorname{der}(A, M)$. Thus the direct sum $M^{l} \oplus R \operatorname{der}$ $(A, M)$ is all of $\mathscr{D}_{A}^{1}(A, M)$.

Part (a, ii) shows how right derivations from $A$ to $M$ give rise to inner derivations from $A$ to $\operatorname{Hom}(A, M)$. These inner derivations are characterized in the next proposition.

For $f: A \rightarrow \operatorname{Hom}(A, M)$ let $f^{(1)}: A \rightarrow M$ be defined by

$$
f^{(1)}(a) \equiv f(a)(1) \in M
$$

i.e., $f(a)$ evaluated at 1 .

Proposition 5.7. (a) Suppose $d: A \rightarrow \operatorname{Hom}(A, M)$ is a derivation. The following conditions are equivalent:

(i ) $d(A) \subset M^{l}$

(ii) $d=d^{(1) l}$

(iii) $d^{(1)}: A \rightarrow M$ is a right derivation.

(b) All derivations from $A$ to $\operatorname{Hom}(A, M)$ are inner. If $d: A \rightarrow \operatorname{Hom}(A, M)$ is a derivation then $d=\delta d^{(1)}$.

Proof. (i) $\Leftrightarrow$ (ii). Since $M \stackrel{l}{\rightarrow} \operatorname{Hom}(A, M)$ is injective if $d(A) \subset M^{l}$ there is a unique $t: A \rightarrow M$ with $d=t^{l}$. For $a \in A$ evaluate $d(a)=$ $t^{l}(a)$ at $1 \in A$ to conclude $t=d^{(1)}$. The converse is clear.

(ii) $\Rightarrow$ (iii). By the equivalence of (i) and (iv) in $(5.4$, a) and since $d=d^{(1) l}$ is a derivation it follows that $d^{(1)}$ is a right derivation. tion

(iii) $\Rightarrow$ (ii). Suppose $a, b \in A$, then since $d^{(1)}$ is a right deriva-

$$
\begin{aligned}
& \quad \|^{(1)}(a b)=\underbrace{d^{(1)}(a) \cdot b+d^{(1)}(b) \cdot a}_{\|} \\
& \quad \overbrace{(a b)(1) \quad d(a)(1) \cdot b+d(b)(1)}^{\|} \cdot a \\
& \quad \text { since } d \text { is a derivation } \\
& (a \cdot d(b)+d(a) \cdot b)(1)=d(b)(1 a)+d(a)(1) \cdot b .
\end{aligned}
$$

Subtracting the common term $d(a)(\mathbf{1}) \cdot b$ leaves

$$
d(b)(a)=d(b)(1) \cdot a
$$

or

$$
d(b)(a)=d^{(1) l}(b)(a)
$$

Hence $d=d^{(1) l}$. 
(b) If $d: A \rightarrow \operatorname{Hom}(A, M)$ is a derivation then $d(a b)-d(a) \cdot b=$ $a \cdot d(b)$. Evaluating at 1 shows

$$
\overbrace{\left(b \cdot d^{(1)}-d^{(1)} \cdot b\right)(a)}^{d(a b)(1)-d(a)(1)} \cdot b=d(b)(1 a)
$$

or

$$
\delta d^{(1)}(b)(a)=d(b)(a)
$$

Hence $d=\delta d^{(1)}$.

As mentioned earlier there is a Kähler module for right derivations which we now develop.

Recall (1.16) $\left(J_{1}(A), j_{1}\right)$. The map $j_{1}: A \rightarrow J_{1}(A)$ is defined by the commutative diagram

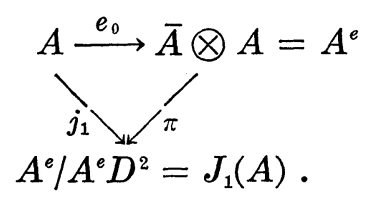

Since $A^{e} D^{2} \subset A^{e} D=\operatorname{Ker} \mu$, (1.9), (1.11), there is a unique factoring $\tilde{\mu}$ of $\mu$ which fits in the commutative diagram with exact rows and columns

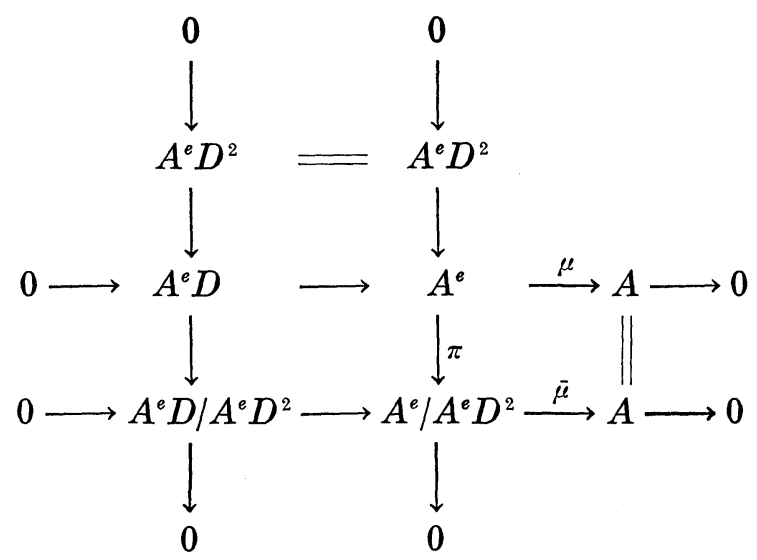

$A^{e}$ has the left $\bar{A}$-module structure arising from $\bar{A} \stackrel{e_{1}}{\rightarrow} A^{e}$. This induces the left $\bar{A}$-module structure on $A^{e} D^{n}$ and $A^{e} / A^{e} D^{n}$ for various $n$. $A$ has the left $\bar{A}$-module structure arising from the right $A_{A}$ structure. Then the maps $\pi, \mu$ and $\tilde{\mu}$ are $\bar{A}$-module maps. The map $\underline{e}_{1}: A \stackrel{\left(a \rightarrow e_{1}(\bar{a})\right)}{\longrightarrow} A^{e}$ is a left $\bar{A}$-module splitting of $\mu$. Hence $\pi \underline{e}_{1}$ is a left $\bar{A}$-module splitting of $\tilde{\mu}$. This shows 


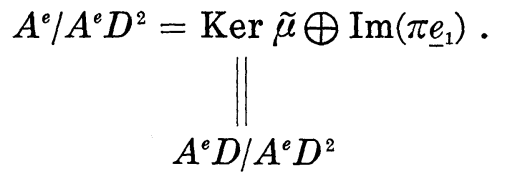

$\underline{e}_{1}(A)=e_{1}(A)$ in fact $A \rightrightarrows \bar{A}$ is a left $\bar{A}$-module isomorphism making the diagram commute

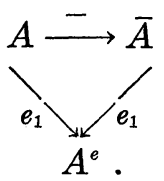

Moreover $\pi e_{1}$ is injective since the composite is $\tilde{\mu} \pi e_{1}: \bar{A} \rightarrow A, \bar{a} \rightarrow a$. Thus we have

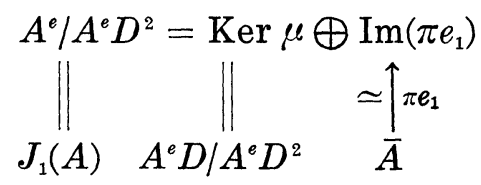

a direct sum decomposition of left $\bar{A}$-modules. It is simple to check that the map $j_{1}: A \rightarrow J_{1}(A)$ breaks up

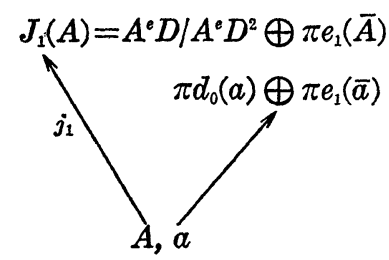

with $d_{0}$ defined in (1.7) by

$$
\overline{1} \otimes a-\bar{a} \otimes 1=d_{0}(a) \in A^{e} .
$$

Kähler module definition 5.11. Let $K_{A}=A^{e} D / A^{e} D^{2}$ and $\partial: A \rightarrow K_{A}$ is $\pi d_{0}$.

$\left(K_{A}, \partial\right)$ is universal for right derivations as we soon indicate. $\left(K_{A}, \partial\right)$ inherits may properties from $J_{1}(A)$ such as behavior with respect to algebra maps $A \stackrel{r}{\rightarrow} B$ and commuting with base ring extension. Note that $K_{A}$ is generated by $\partial(A)$ as a left $\bar{A}$-module (or right $A$-module). Most of the proof of the next theorem is left to the reader.

THEOREM 5.12.

(a) Universal property. $\partial: A \rightarrow K_{A}$ is a right derivation. If 
$N$ is any right $A$-module and $d \in R \operatorname{der}(A, N)$ then there is a unique $g \in \operatorname{Hom}_{A}\left(K_{A}, N\right)$ with $g \partial=d$. Thus the map

$$
\begin{aligned}
\operatorname{Hom}_{A}(K, N) & \longrightarrow R \operatorname{der}(A, N) \\
g & \longrightarrow g \partial
\end{aligned}
$$

is bijective.

(b) Relation to defferential separability. The following conditions are equivalent

(i) $A$ is differentially separable

(ii) $K_{A}=\{0\}$

(iii) For all right $A$-modules $N$,

$$
R \operatorname{der}(A, N)=\{0\} .
$$

(c) Algebra map behavior. Say $\gamma: A \rightarrow B$ is an algebra map then there is a unique map $\gamma^{K} \in \operatorname{Hom}_{A}\left(K_{A}, K_{B}\right)$ with the property $\gamma^{K} \partial_{A}=\partial_{B} \gamma$. If $\gamma$ is surjective so is $\gamma^{K}$.

(d) Base change behavior. If $S$ is a commutative $R$-algebra then there is a natural isomorphism $S \otimes K_{A} \stackrel{\cong}{\rightarrow} K_{S \otimes A}^{S}$ of left $S \otimes \bar{A}$ modules making the diagram commute

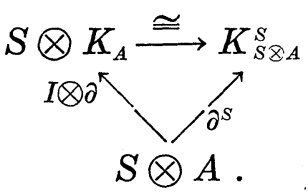

(e) If $A$ is a commutative algebra then $\left(K_{A}, \partial\right)$ is the ordinary Kähler module and universal derivation for $A$.

Proof. (a) Consider $K_{A} \subset J_{1}(A)$ then $\partial=j_{1}-\pi \underline{e}_{1}$. Since $j_{1} \epsilon$ $\mathscr{D}_{A}^{1}\left(A, J_{1}(A)\right)$ and $\pi e_{1} \in \operatorname{Hom}_{\bar{A}}\left(A, J_{1}(A)\right)=\mathscr{D}_{A}^{0}\left(A, J_{1}(A)\right) \subset \mathscr{D}_{A}^{1}\left(A, J_{1}(A)\right)$ it follows that $\partial \in \mathscr{D}_{A}{ }^{1}\left(A, J_{1}(A)\right)$, so of course $\partial \in \mathscr{D}_{A}{ }^{1}\left(A, K_{A}\right)$. However, $\partial(1)=0$ so by $(5.4$, a, $(\mathrm{i}) \Leftrightarrow(\mathrm{v})) \partial$ is a right derivation. The other claims in (a) follow from (5.4) and use of the universal property of $\left(J_{1}(A), j_{1}\right)$.

(b) See $(1.21$, a, (i) $\Leftrightarrow$ (ii)).

(c) See remarks above (3.2) and $(3.2, \mathrm{c}, \mathrm{i})$.

(d) See (4.4).

(e) See $[5,(1.5)$, p. 223].

The next theorem concerns the problem of inducing right derivations. Suppose $V$ is an $R$-submodule of $A, M$ a right $A$-module and $f: V \rightarrow M$ an $R$-module map. When does $f$ extend to a right derivation from $A$ to $M$. The answer comes down to the induction of usual derivations plus $(5.4, \mathrm{~b}$, (ii)). 
EXTENSION THEOREM 5.13. Suppose the algebra $A$ is generated as an algebra by the set $\mathscr{S}$. Let $M$ be a right $A$-module.

(a) If $E, F: A \rightarrow M$ are right derivations and $E|\mathscr{S}=F| \mathscr{S}$ then $E=F$.

(b) If $g: \mathscr{S} \rightarrow M$ is a set map satisfying

$$
(*) \quad 0=\sum_{i=1}^{n} g\left(s_{i}\right)\left[\left(s_{1} \cdots \widehat{s_{i}} \cdots s_{n}\right), u\right]
$$

for $2 \leqq n \in Z, \quad s_{1}, s_{2}, \cdots, s_{n} \in \mathscr{S}, u \in A . \quad$ Let $g^{l}: \mathscr{S} \rightarrow \operatorname{Hom}(A, M)$, $s \rightarrow g(s)^{l}$. If there is a derivation $d: A \rightarrow \operatorname{Hom}(A, M)$ extending $g^{l}$ then there is a unique right derivation $G: A \rightarrow M$ extending $g$. Moreover $d=G^{l}$.

Proof. (a) $E-F: A \rightarrow M$ is a right derivation with $\mathscr{S} \in \mathrm{Ker}$ $(E-F)$. By $(5.4,(\mathrm{~b}),(\mathrm{iv})) \operatorname{Ker}(E-F)$ is a subalgebra of $A$. Since $\mathscr{S}$ generates $A$ it follows $A=\operatorname{Ker}(E-F)$ so $E=F$.

(b) Uniqueness of $G$ follows from part (a). Suppose $d: A \rightarrow$ $\operatorname{Hom}(A, M)$ is a derivation extending $g^{l}$, we show $d(A) \subset M^{l} . d(1) 0=$ $0 \in M^{l}$, and for $s \in \mathscr{S} d(s)=g(s)^{l} \in M^{l}$. Thus monomials of length 0 or 1 of elements of $\mathscr{S}$ are mapped to $M^{l}$ by $d$. Say $2 \leqq n \in Z$ and $s_{1}, \cdots, s_{n} \in \mathscr{S}$. We shall show that $d\left(s_{1} \cdots s_{n}\right) \in \operatorname{Hom}_{A}(A, M)=M^{l}$.

Since $d$ is a derivation $d\left(s_{1} \cdots s_{n}\right)=\sum_{i=1}^{n} s_{1} \cdots s_{i-1} \cdot d\left(s_{i}\right) s_{i+1} \cdots s_{n}$ and $d\left(s_{i}\right)=g\left(s_{i}\right)^{l}$. Thus for $a, c \in A$

$$
\begin{aligned}
d\left(s_{1}\right. & \left.\cdots s_{n}\right)(c a) \\
& =\left(\sum_{i=1}^{n} s_{1} \cdots s_{i-1} \cdot g\left(s_{i}\right)^{l} \cdot s_{i+1} \cdots s_{n}\right)(c a) \\
& =\sum_{i=1}^{n}\left(g\left(s_{i}\right) \cdot\left[(c a) s_{1} \cdots s_{i-1}\right]\right) \cdot\left(s_{i+1} \cdots s\right) \\
& =\sum_{i=1}^{n} g\left(s_{i}\right) \cdot(c a) \cdot\left(s_{1} \cdots \widehat{s_{i}} \cdots s_{n}\right)
\end{aligned}
$$

by $(*)$

$$
\begin{aligned}
& =\sum_{i=1}^{n} g\left(s_{i}\right) \cdot\left(s_{1} \ldots \widehat{s_{i}} \cdots s_{n}\right) \cdot(c a) \\
& =\left(\sum_{i=1}^{n} g\left(s_{i}\right) \cdot\left(s_{1} \cdots \widehat{s_{i}} \cdots s_{n}\right) \cdot c\right) \cdot a
\end{aligned}
$$

by $(*)$

$$
=\left(\sum_{i=1}^{n} g\left(s_{i}\right) \cdot c \cdot\left(s_{1} \cdots \widehat{s_{i}} \cdots s_{n}\right)\right) \cdot a
$$

similarly to above

$$
=\left(d\left(s_{1} \cdots s_{n}\right)(c)\right) \cdot a .
$$

Hence $d$ carries all length momonoials of elements of $\mathscr{S}$ to $M^{\imath}$. 
Since $\mathscr{S}$ generates $A$ it follows $d(A) \subset M^{l}$. By $(5.7$, (i) $\Leftrightarrow$ (ii)) $d^{(1)}: A \rightarrow M$ is a right derivation. For $s \in \mathscr{S}, d^{(1)}(s)=g(s)$.

(Finite) Generation Theorem 5.14. Suppose the algebra $A$ contains subsets $\mathscr{S}$ and $\mathscr{S}$ satisfying the following conditions:

(i) $A$ is generated as an algebra by $\mathscr{S} \cup \mathscr{S}$.

(ii) The elements of $\mathscr{I}$ are invertible in $A$ with the inverses lying in the subalgebra of $A$ generated by $\mathscr{S}$.

If $g: A \rightarrow M$ is a right derivation then $g(A) A=g(\mathscr{S}) A$. In particular if $\mathscr{S}$ is a finite set then $g(A) A$ is a finitely generated A-module.

Proof. Let $M^{\prime}=M / g(\mathscr{S}) A$ and let $g^{\prime}$ be the right derivation $A \stackrel{g}{\rightarrow} M \rightarrow M / g(\mathscr{S}) A=M^{\prime}$. Ker $g^{\prime}$ is a subalgebra of $A$ by $(5.4, \mathrm{~b}$, vi) and $\mathscr{S} \subset \operatorname{Ker} g^{\prime}$ so that $S$ the subalgebra of $A$ generated by $\mathscr{S}$ lies in $\operatorname{Ker} g^{\prime}$. For $x \in d$ by hypothesis $x^{-1} \in S \subset \operatorname{Ker} g^{\prime}$ and by (5.4, b, vi) $\mathscr{I} \subset \operatorname{Ker} g^{\prime}$. Since $\mathscr{I} \cup \mathscr{S}$ generates $A$ as an algebra it follows that $A \subset \operatorname{Ker} g^{\prime}$ which implies $g(A) \subset g(\mathscr{S}) A$. Hence $g(\mathscr{S}) A \subset g(A) A \Subset$ $g(\mathscr{S}) A$.

Definition 5.15. If $g: A \rightarrow M$ is a right derivation then

$$
\mathscr{C}_{g}=\{x \in A \mid x A \subset \operatorname{Ker} g\}
$$

is called the conductor of $g$.

Recall $(5.4, \mathrm{~b}, \mathrm{vi})$ that $\operatorname{Ker} g$ is a subalgebra of $A$.

DeCOMPOSITION THEOREM 5.16.

(a) (i) $\mathscr{C}_{g}=\{x \in A \mid A x \subset \operatorname{Ker} g\}$.

(ii) $\mathscr{C}_{g} \subset \operatorname{Ker} g$ and $\mathscr{C}_{g}$ is the unique maximal left (right, twosided) ideal in Ker $g$ which is also an ideal in $A$.

(iii) $(g(A) \cdot A) \cdot \mathscr{C}_{g}=\{0\}$.

Replace $M$ by the submodule $M^{\prime}=g(A) \cdot A$ which by (iii) is a module for $A^{\prime}=A / \mathscr{C}_{g}$. Since $\mathscr{C}_{g} \subset \operatorname{Ker} g, g$ factors

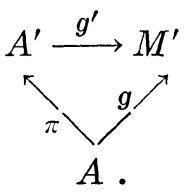

(b) $g^{\prime}$ is a right derivation with same image as $g$. In particular $g=0$ if and only if $g^{\prime}=0$.

(c) $A^{\prime}$ has the properties:

(i) for $0 \neq a \in \operatorname{Ker} g^{\prime}$ there is $b \in A^{\prime}$ with $b a$, $a b \notin \operatorname{Ker} g^{\prime}$.

(ii) $\left[A^{\prime}, A^{\prime}\right] \subset \operatorname{Ker} g^{\prime}$. 
(iii) $\operatorname{Ker} g^{\prime}$ is central in $A^{\prime}$.

(iv) $\left[A^{\prime}, A^{\prime}\right]$ lies in the Jacobson radical of $A^{\prime}$; equivalently, mod any maximal ideal $A^{\prime}$ is a field.

(v) If $\left[A^{\prime}, A^{\prime}\right]$ is a left (right) ideal in $A^{\prime}$ then $\left[A^{\prime}, A^{\prime}\right]=\{0\}$ so that $A^{\prime}$ is commutative.

(d) If $A$ is semi-simple then $A^{\prime}$ is commutative.

Proof. (a) (i) follows from $g(x a)=g(a x),(5.4, \mathrm{~b}, \mathrm{i})$.

(ii) For $x \in \mathscr{C}_{g} g(x)=g(1 x) \in \operatorname{Ker} g$, so that $\mathscr{C}_{g} \subset \operatorname{Ker} g$. For $a, u \in A$

$$
(x u) a=x(u a) \in \operatorname{Ker} g
$$

and $x u \in \mathscr{C}_{g}$. To show $u x \in \mathscr{C}_{g}$ we use (i) so

$$
a(u x)=(a u) x \in \operatorname{Ker} g
$$

and $\mathscr{C}_{g}$ is a 2 -sided ideal in $A$. It is easily verified from the definition of $\mathscr{C}_{g}$ that $\mathscr{C}_{g}$ is the unique maximal right ideal of $\mathscr{C}_{g}$ which is a right ideal in $A$. By (i) the same holds with "right" replaced by "left". Hence, it is true with "right" and "left" replaced by "two-sided".

(iii) Since $\mathscr{C}_{g}$ is an ideal in $A$

$$
g(A) A \mathscr{C}_{g}=g(A) \mathscr{C}_{g}
$$

and it suffices to show $g(A) \mathscr{C}_{g}=\{0\}$. For $a \in A, x \in \mathscr{C}_{g}$

$$
g(a) x=\underbrace{g(a x)}_{\in}-g \underbrace{g(x)}_{\substack{\epsilon \\ \operatorname{Ker} g}} a=0
$$

(b) is trivial and left to the reader.

(c) (i) $K=\left\{a \in \operatorname{Ker} g^{\prime} \mid b a \in \operatorname{Ker} g^{\prime}\right.$ for all $\left.b \in A\right\}$ is a left ideal in Ker $g^{\prime}$ which is a left ideal in $A^{\prime}$. Since $\pi(\operatorname{Ker} g)=\operatorname{Ker} g^{\prime}$ it follows from (a, ii) that $K=\{0\}$. Hence for $0 \neq a \in \operatorname{Ker} g^{\prime}$ there is $b \in A^{\prime}$ with $g^{\prime}(b a) \neq 0$. Since $g^{\prime}(a b)=g^{\prime}(b a)$ it follows that $a b \notin \operatorname{Ker} g^{\prime}$.

(ii) This is immediate from

$$
g(b a)=g(a b), \quad(5.4, \mathrm{~b}, \mathrm{i}) .
$$

(iii) From $\left(5.4, \mathrm{~b}\right.$, ii) for $a, b, c \in A^{\prime}$

$$
g(a) \cdot[b, c]+g(b) \cdot[a, c]=0 .
$$

If $a \in \operatorname{Ker} g^{\prime}$ this gives

$$
g^{\prime}(b) \cdot[a, c]=0 \quad \text { for } \quad a \in \operatorname{Ker} g^{\prime}, \quad b, c \in A^{\prime}
$$

and by $\left(5.4, \mathrm{~b}\right.$, vii) $g^{\prime}$ is $\left(\operatorname{Ker} g^{\prime}\right)$-linear so that 


$$
g^{\prime}(b[a, c])=0 \text { for } a \in \operatorname{Ker} g^{\prime}, \quad b, c \in A^{\prime} .
$$

If $[a, c]$ were nonzero (and since $[a, c] \in \operatorname{Ker} g^{\prime}$ by $(c, i)$ ) for some $b \in A^{\prime}, b[a, c] \notin \operatorname{Ker} g^{\prime}$ which contradicts $\left(^{*}\right)$. Hence $\left[\operatorname{Ker} g^{\prime}, A^{\prime}\right]=\{0\}$ and $\operatorname{Ker} g^{\prime}$ is central in $A^{\prime}$.

(iv) If $\mathscr{C}$ is a maximal ideal of $A^{\prime}$ then $A^{\prime} / \mathscr{C}$ is simple. By (c, ii and iii) $\left[A^{\prime}, A^{\prime}\right]$ is central in $A^{\prime}$ so that $\left[A^{\prime} / \mathscr{M}, A^{\prime} / \mathscr{M}\right]$ is central in $A^{\prime} / \mathscr{C}$. By the next lemma (5.21) $A^{\prime} / \mathscr{C}$ is a field.

(v) follows from (c, i and ii).

(d) If $A$ is semi-simple then $A$ and hence $A^{\prime}$ have Jacobson radical zero. By (c, iv) this implies that $A^{\prime}$ is commutative.

In particular it follows from part (d) that if $A$ is simple and has a nonzero right derivation then $A$ must be commutative; hence a field. This may be stated as

COROLlary 5.19. If $A$ is a simple algebra which is not commutative then $A$ is right differentially separable.

Corollary 5.20. If $K_{A}$ is a faithful right A-module (true if $A$ is simple and $\left.K_{A} \neq\{0\}\right)$ then

(i) $[A, A] \subset \operatorname{Ker} \partial$ and $\operatorname{Ker} \partial$ is central in $A$.

(ii) If $J$ is the Jacobson radical of $A$ then $A / J$ is commutative. In particular $A$ is commutative if semi-simple.

(iii) If $J$ is a nil ideal i.e., consists of nilpotent elements then $A$ is commutative if $\operatorname{Ker} \partial$ (or the center of $A$ ) is semi-simple.

Proof. Use $K_{A}$ for $M$ and $\partial$ for $g$ in (5.16). By the remark above (5.12), $K_{A}=\partial(A) A$ so that $K_{A}=M^{\prime}$. Since $K_{A}$ is assumed to be a faithful right $A$-module, $\left(5.16\right.$, a, iii) implies that $\mathscr{C}_{\partial}=\{0\}$. Hence $A=A^{\prime}$. Then (i) follows from (5.16, c, ii and iii). And (ii) follows from $(5.16, \mathrm{c}, \mathrm{iv})$ which insures $[A, A] \subset J$.

If $J$ is a nil ideal then the intersection $J \cap \operatorname{Ker} \partial$ (or $J \cap$ (center of $A)$ ) lies in the radical of the subalgebra. Semi-simplicity then insures that the intersection is zero. Since $[A, A]$ lies in the intersection we have that $A$ is commutative, proving (iii).

Lemma 5.21. If $U$ is a simple ring with center $C$ and $[U, U] \subset C$ then $U=C$ so that $U$ is a field.

Proof. It is well known (or easily verified) that $C$ is a field. Suppose there are elements $u, v \in U$ with $0 \neq[u, v]$. By hypothesis $[u, v] \in C$, a field, so that there is $c \in C$ with $1=[u, v] c$. Since $C$ is central $[u, v c]=[u, v] c=1$. Replacing $v$ by $v c$ we may assume $[u, v]=1$. For any $x \in U$ 


$$
x=[u, v] x=[u, v x]-v[u, x]
$$

so that as a vector space over $C, U$ is spanned by 1 and $v$. Hence $\operatorname{dim}_{C} U \leqq 2$. By a Wedderburn structure theorem $\operatorname{dim}_{C} U$ must be a square when finite. Hence it follows that $\operatorname{dim}_{C} U$ must be 1 .

6. Right differentially separable algebras.

Proposition 6.1. Consider the conditions:

(i) $A$ is separable,

(ii) $\left(A^{e} D\right)^{2}=A^{e} D$,

(iii) $A$ is right differentially separable.

(a) $\quad$ (i) $\Rightarrow$ (ii),

(b) If $R$ is semi-simple then (i) implies (iii),

(c) If $A$ is commutative (ii) is equivalent to (iii).

(d) If $A$ is commutative and $A^{e} D$ is a finintely generated ideal (true if $A^{e}$ is Noetherian) then (i) and (ii) and (iii) are equivalent.

Proof. Separability of $A$ is equivalent to $A^{e} D$ beidg generated as a left ideal by an idempotent element. We briefly sketch a proof of this equivalence. The proof of the proposition begins in ernest below (6.2).

A being separable is equivalent to $A$ being a projective $A^{e}$-module. This is equivalent to the existence of a splitting (as left $A^{e}$-modules) to the map $A^{e} \stackrel{\mu}{\rightarrow} A$. Hence

A splitting $\sigma$ satisfies $\sigma(a) \cdot 1=a$ since $z \cdot 1=\mu(z)$ for $z \in A^{e}$.

$$
\sigma(1) \cdot \sigma(1)=\sigma(\sigma(1) \cdot 1)=\sigma(\mu \sigma(1))=\sigma(1)
$$

and $\sigma(1)$ is idempotent. Moreover $A^{e} D \cdot \sigma(1)=\sigma\left(A^{e} D \cdot 1\right)=\{0\} . \quad$ It is easily shown that given $u \in A^{e}$ with $A^{e} D \cdot u=\{0\}$ and $\mu(u)=1$ then $\sigma_{u}: A \rightarrow A^{e}, a \rightarrow e_{0}(a) u=e_{1}(\bar{a}) u$ is a splitting of $\mu$ as left $A^{e}$-modules. Hence $u=\sigma_{u}(1)$ is idempotent as is $e=1-u$. Since $A^{e} D \cdot u=\{0\}$ and $e+u=1$ it follows that $e$ is an idempotent generator of the left ideal $A^{e} D$. If $f$ is an idempotent generator of $A^{e} D$ then $\mu(1-f)=1$ since $A^{e} D=\operatorname{ker} \mu\left(1.11\right.$, a) and $A^{e} D \cdot(1-f)=\{0\}$. Hence we have a bijective correspondence between idempotent generators of $A^{e} D$ and $A^{e}$-module splittings of $\mu$ given by $e \rightarrow \sigma_{1-e}$. In particular:

$$
\left\{\begin{array}{l}
A \text { is a separable algebra if and only if } \\
\text { the left ideal } A^{e} D \text { has an idempotent } \\
\text { generator. }
\end{array}\right.
$$


(a) If $A^{e} D$ has an idempotent generator $e$ then $\left(A^{e} D\right)^{2} \supset A^{e} D e=$ $A^{e} D$ and (i) implies (ii).

(c) If $A$ is commutative then so is $A^{e}$ and $A^{e} D^{2}=\left(A^{e} D\right)^{2}$. Thus $A^{e} D^{2}=A^{e} D$ if and only if $\left(A^{e} D\right)^{2}=A^{e} D$.

(d) If $A$ and hence $A^{e}$ are commutative and $A^{e} D$ is a finitely generated ideal equal to its own square then by [4, Theorem $76, \mathrm{p}$. 50], $A^{e} D$ has an idempotent generator. Thus by (6.2) $A$ is separable and (ii) implies (i). We have already shown in (a) that (i) implies (ii) and in (c) that (ii) and (iii) are equivalent.

(b) Suppose $A$ is separable and $R$ is semi-simple. We show that all right derivations of $A$ are trivial so that $A$ is right differentially separable by $(5.12, \mathrm{~b})$. We must use (a) and (c) which have already been established.

Suppose $M$ is a right $A$-module and $g: A \rightarrow M$ a right derivation. Use the decomposition theorem (5.16) to obtain the factorization (5.17)

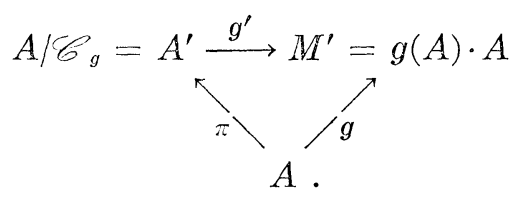

To show $g$ is zero it suffices to show that $g^{\prime}$ is zero since they have the same image. Since $A$ is separable so is $A / \mathscr{C}_{g}$. Since $R$ is semi-simple, $A / \mathscr{C}_{g}$ has radical zero by [2, Proposition 7.6, p. 179] Hence by $\left(5.16\right.$, c, iv) $A / \mathscr{C}_{g}$ is commutative, as well as separable. Hence by (a) and (c) applied to $A / \mathscr{C}_{g}$ it follows that $A / \mathscr{C}_{g}$ is right differentially separable. Thus $g^{\prime}=0$.

The commutator ideal $A[A, A] A$ of $A$ is denoted $\%$. Since $[a, b] c=[a, b c]-b[a, c]$ it follows that $[A, A] A \subset A[A, A]$. The opposite inclusion is similar so that

$$
\mathscr{K}=A[A, A]=[A, A] A .
$$

Right Differential Separability Theorem 6.3. (a) If $A$ is right differentially separable then $A / \mathscr{K}$ is right differentially separable.

(b) If $K_{A}$ is a finitely generated A-module (see (5.14) for conditions) and $A / \mathscr{K}$ is right differentially separable then $A$ is right differentially separable (so that actually $K_{A}=\{0\}$ ).

(c) Suppose $A$ contains subsets $\mathscr{I}$ and $\mathscr{S}$ satisfying:

(i) $A$ is generated as an algebra by $\mathscr{F} \cup \mathscr{S}$.

(ii) The elements of $\mathscr{I}$ are invertible in $A$ with the inverses lying in the subalgebras of $A$ generated by $\mathscr{S}$. 
(iii) $\mathscr{S}$ is a finite set.

Then $A$ is right differentially separable if $A / \mathscr{K}$ is a separable algebra. And if $R$ is Noetherian, A/ K is a separable algebra if $A$ is differentially separable. In particular, finitely generated algebras over Noetherian rings are right differentially separable if and only if "abelianized" they are separable.

Proof. (a) is a special case of $(3.2, \mathrm{c}, \mathrm{ii})$.

(b) Suppose $A$ is not right differentially separable. By (5.12, b) $K_{A} \neq\{0\}$ and as observed above (5.12) $K_{A}$ is generated by $\partial(A)$ as right $A$-module. Thus $0 \neq \partial$. We shall show that $A / \mathscr{C}$ is not right differentially separable by producing a commutative quotient algebra $B$ of $A$ and nonzero right derivation $B \stackrel{g}{\rightarrow} M$. Since $\mathscr{C}$ is the commutator ideal of $A$ the quotient $A \rightarrow B$ factors $A \rightarrow A / \mathscr{K} \rightarrow B$. Since $B$ is not right differentially separable by $(3.2$, c, ii) $A / \mathscr{K}$ is not right differentially separable.

We apply the Decomposition Theorem (5.16) to $\partial: A \rightarrow K_{A}=$ $\partial(A) \cdot A$, to obtain the factorization:

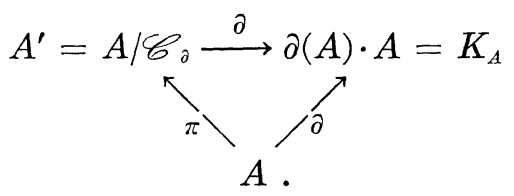

The $A^{\prime}$-module structure on $K_{A}$ arises from the $A$-module structure so that $K_{A}$ is a finitely generated right $A^{\prime}$-module. Also $0 \neq \partial^{\prime}$ since $\partial^{\prime}\left(A^{\prime}\right) \cdot A^{\prime}=\partial(A) \cdot A=K_{A} \neq\{0\}$. By (5.16, c, iv), $\left[A^{\prime}, A^{\prime}\right]$ lies in the Jacobson radical of $A^{\prime}$. Let $\mathscr{K}^{\prime}$ be $A^{\prime}\left[A^{\prime}, A^{\prime}\right] A^{\prime}$, the commutator ideal of $A^{\prime}$. So $\mathscr{K}^{\prime}$ lies in the Jacobson radical of $A^{\prime}$. By the Nakayama lemma [4, Theorem 28, p. 51] it follows that $K_{A} \cdot \mathscr{K}^{\prime} \nsubseteq K_{A}$. Let $M$ be the quotient $A^{\prime}$-module $K_{A} / K_{A} \mathscr{K}^{\prime} \neq\{0\}$.

As observed above (6.3), $\mathscr{K}^{\prime}=A^{\prime}\left[A^{\prime}, A^{\prime}\right]$. Let $u, v, w \in A^{\prime}$, then $\partial^{\prime}(u[v, w])=\partial^{\prime}(u) \cdot[v, w]$ by $\left(5.4, \mathrm{~b}\right.$, vii). Thus $\partial^{\prime}\left(\mathscr{K}^{\prime}\right) \subset K_{A} / K_{A} \mathscr{K}^{\prime}$ and $\partial^{\prime}$ factros through the commutative algebra $A^{\prime} / \mathscr{K}^{\prime}=B$ as indicated in the diagram:

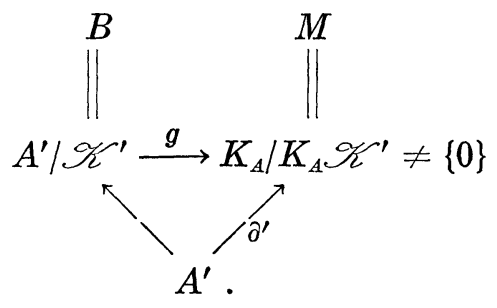

Since $M \cdot \mathscr{K}^{\prime}=\{0\}$ the right $A^{\prime}$-module structure on $M$ induces 
a right $B$-module structure on $M$. It is easily checked and left to the reader that $g: B \rightarrow M$ is a right derivation. Since $K_{A}$ is generated by $\partial^{\prime}\left(A^{\prime}\right)$ as a right $A^{\prime}$-module, $M \neq\{0\}$ is generated by $g(B)$ as a right $B$-module. Hence, $g \neq 0$, proving (b).

(c) If $A / \mathscr{K}$ is separable by $(6.1, \mathrm{a}, \mathrm{c}) A / \mathscr{K}$ is right differentially separable. By (5.14) the hypotheses governing $\mathscr{J}$ and $\mathscr{S}$ insure that $K_{A}=\partial(A) \cdot A$ is a finitely generated $A$-module. Hence by part (b) of this theorem $A$ is right differentially separable.

Conversely suppose $A$ is right differentially separable and $R$ is Noetherian. By part (a) of this theorem $A / \mathscr{K}$ is right differentially separable. Let $\mathscr{I}^{\prime}$ and $\mathscr{S}^{\prime}$ be the images of $\mathscr{J}$ and $\mathscr{S}$ respectively in the commutative algebra $A / \mathscr{K}$. The subalgebra $X$ of $(A / \mathscr{K})^{e}$ generated by the finite set

$$
\{1 \otimes s\}_{s \in \mathscr{S}} \cup\{t \otimes 1\}_{t \in \mathscr{S}} .
$$

is Noetherian by the Hilbert basis theorem [4, Theorem 69, p. 47] and a bit of fiddling. (We are identifying $A / \mathscr{K}$ with $\overline{A / \mathscr{K}}$ and $(A / \mathscr{K})^{e}$ with $A / \mathscr{K} \otimes A / \mathscr{K}$ since $A / \mathscr{K}$ is commutative). Let $S$ be the multiplicative system in $(A / \mathscr{K})^{e}$ generated by elements of the set

$$
\{u \otimes 1\}_{u^{-1} \in \mathscr{I}} \cup\{1 \otimes v\}_{v^{-1} \in \mathscr{J}^{\prime}} .
$$

The condition that $A$ is generated by $\mathscr{I} \cup \mathscr{S}$ implies that $(A / \mathscr{K})^{e}$ is $X$ localized at $S$; hence, by [4, Theorem 85, p. 57] $(A / \mathscr{K})^{e}$ is Noetherian. Now $(6.1, \mathrm{c}, \mathrm{d})$ implies that $A / \mathscr{K}$ is separable.

\section{REFERENCES}

1. N. Bourbaki, Elements de Mathematique, Algebre commutative, Hermann Chap. 1.

2. H. Cartan and S. Eilenberg, Homological Algebra, Princeton University Press, Princeton, New Jersey, 1956.

3. R. Heyneman and M. Sweedler, Affine Hopf algebras, I, J. Algebra, 13 (1969), 192-241.

4. I. Kaplansky, Commutative Rings, Allyn and Bacon, Inc., Boston, Mass., 1970.

5. H. Osborn, Modules of differentials, I. Math. Ann., 170 (1967), 221-244.

6. M. E. Sweedler, Nilpotent and solvable associative algebras, J. Algebra, 28 (1974), 102-110.

7. M. E. Sweedler, Purely inseparable algebras, J. Algebra, 35 (1975), 342-355.

Received May 1, 1978. Supported in part by NSF MCS 73-04876.

CORNELL UNIVERSITY

ITHACA, NY 14853 


\section{PACIFIC JOURNAL OF MATHEMATICS}

\section{EDITORS}

DONALD BABBITT (Managing Editor)

University of Galifornia

Los Angeles, California 90024

HUGo RossI

University of Utah

Salt Lake City, UT 84112

C. C. MOORE AND ANDREW OGG

University of California

Berkeley, CA 94720
J. DUGUNDJI

Department of Mathematics University of Southern California Los Angeles, California 90007

R. FINN AND J. MILGRAM Stanford University Stanford, California 94305

\section{ASSOCIATE EDITORS}

E. F. BECKENBACH

B. H. NEUMANN

F. WOLF

K. YosHIDA

\section{SUPPORTING INSTITUTIONS}

UNIVERSITY OF BRITISH COLUMBIA CALIFORNIA INSTITUTE OF TECHNOLOGY UNIVERSITY OF CALIFORNIA MONTANA STATE UNIVERSITY UNIVERSITY OF NEVADA, RENO NEW MEXICO STATE UNIVERSITY OREGON STATE UNIVERSITY UNIVERSITY OF OREGON
UNIVERSITY OF SOUTHERN CALIFONIA STANFORD UNIVERSITY UNIVERSITY OF HAWAII UNIVERSITY OF TOKYO UNIVERSITY OF UTAH WASHINGTON STATE UNIVERSITY UNIVERSITY OF WASHINGTON 


\section{Pacific Journal of Mathematics}

\section{Vol. 86, No. $1 \quad$ November, 1980}

Gert Einar Torsten Almkvist, Invariants, mostly old ones .............. 1

Hyman Bass, Groups of integral representation type ................ 15

A. Białynicki-Birula, On action of SL(2) on complete algebraic

varieties........................................

Frederick Paul Greenleaf and Martin Allen Moskowitz, Groups of

automorphisms of Lie groups: density properties, bounded orbits, and

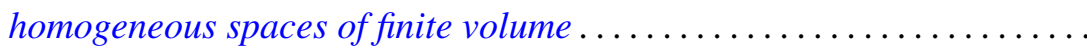

Raymond Taylor Hoobler, A cohomological interpretation of Brauer groups

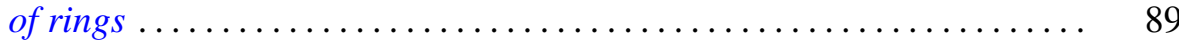

Irving Kaplansky, Superalgebras ........................ 93

Jerrold Lewis Kleinstein and Alex I. Rosenberg, Succinct and

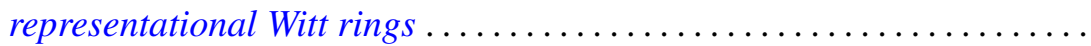

E. R. Kolchin, On universal extensions of differential fields ............ 139

Andy R. Magid, Analytic subgroups of affine algebraic groups. II ....... 145

Calvin Cooper Moore, The Mautner phenomenon for general unitary

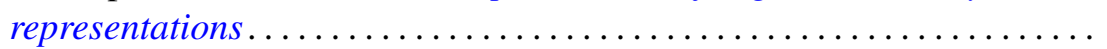

George Daniel Mostow, On a remarkable class of polyhedra in complex hyperbolic space ................................ 171

Brian Lee Peterson, Extensions of pro-affine algebraic groups. II . . . . . . 277

John Henry Reinoehl, Lie algebras and affine algebraic groups......... 287

Maxwell Alexander Rosenlicht, Differential valuations .

John Brendan Sullivan, The second Lie algebra cohomology group and Weyl modules..................................... 321

Moss Eisenberg Sweedler, Right derivations and right differential

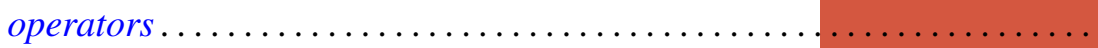

Bostwick Frampton Wyman, Time varying linear discrete-time systems. II. Duality.................................. 